\title{
Update on the Etiology of Tooth Resorption in Domestic Cats
}

\author{
Alexander M. Reiter, Dipl Tzt, Dr Med Vet ${ }^{\mathrm{a}, *}$, \\ John R. Lewis, VMD ${ }^{\mathrm{a}}$, Ayako Okuda, DVM, $\mathrm{PhD}^{\mathrm{b}, \mathrm{c}}$ \\ ${ }^{a}$ Department of Clinical Studies, School of Veterinary Medicine, \\ University of Pennsylvania, 3900 Delancey Street, Philadelphia, PA 19104-6010, USA \\ ${ }^{\mathrm{b}}$ Department of Anatomy, School of Veterinary Medicine, \\ Azabu University, Fuchinobe, Japan \\ ${ }^{\mathrm{c}}$ Vettec Dentistry, Tokyo, Japan
}

Feline odontoclastic resorptive lesions (FORL) were first recognized and histologically differentiated from caries in the 1920s [1,2]. Some anecdotal reports describing caries-like lesions at the cervical region of feline teeth followed in the 1950s and 1960s, until two microscopic studies in the 1970s again revealed that FORL were not caries but a type of tooth resorption $[3,4]$. A recent study showed that cats with FORL have a significantly lower urine specific gravity and significantly higher serum concentration of 25hydroxyvitamin D (25OHD) compared with cats without FORL [5], indicating that multiple tooth resorption in domestic cats could be the manifestation of some systemic insult rather than a local cause. In this article, the histologic and radiographic appearance of FORL and certain other peculiarities of feline teeth are reviewed. An attempt is then made to compare these findings with changes of the periodontium induced by administration of excess vitamin $\mathrm{D}$ or vitamin $\mathrm{D}$ metabolites in experimental animals.

\section{Histologic and radiographic features of feline odontoclastic resorptive lesions}

Tooth resorption is caused by odontoclasts. Their precursors derive from hematopoietic stem cells of bone marrow or spleen and migrate from blood vessels of the alveolar bone or periodontal ligament toward the external root

* Corresponding author.

E-mail address: reiter@vet.upenn.edu (A.M. Reiter). 
surface, where mononuclear cells fuse with other cells to become multinucleated mature odontoclasts [6,7]. One important fact to understand is that FORL develop anywhere on the root surface and not just close to the cementoenamel junction [8]. Resorption of enamel as the initial event is only rarely observed [9]. Resorption may also start on the same tooth at various root surfaces simultaneously, progressing from cementum coronally into crown dentin as well as apically into root dentin. As the resorption progresses into crown dentin, the enamel often becomes undermined and a pink discoloration may be observed at the crown surface [10].

FORL that emerge at the gingival margin were originally referred to as neck lesions (Fig. 1) [4]. Exposure to periodontal inflammation, which is caused and maintained by bacterial infection, results in the formation of highly vascular and inflamed granulation tissue [11-16]. These defects may be painful and bleed easily when probed with a dental instrument [10]. One characteristic feature of inflammatory root resorption is that the alveolar bone adjacent to the tooth defect is also resorbed [17]. Such root lesions have been categorized as type I root lesions if unaffected root areas are surrounded by a radiographically visible periodontal space (Fig. 2) [18]. Although pulp involvement may be seen in advanced stages of FORL $[19,20]$, the cervical root resorption in human beings typically proceeds laterally and in an apical and coronal direction, surrounding a thin shell of dentin and predentin, and envelops the root canal, leaving an apple core appearance of the cervical area of the tooth [21].

It has been demonstrated in several studies in human beings that superficial external resorption is common and usually self-limiting [22]. Spontaneously repaired defects of cementum and superficial dentin are called surface resorptions, in which the anatomic contour of the root surface is restored [17]. Most clinically evident FORL appear histologically to be in resorptive and reparative phases simultaneously [14]. Although attempts at repair can be noted by production of bone, cellular cementum, and bonecementum [12-14,19,20,23], tooth resorption in cats is usually progressive

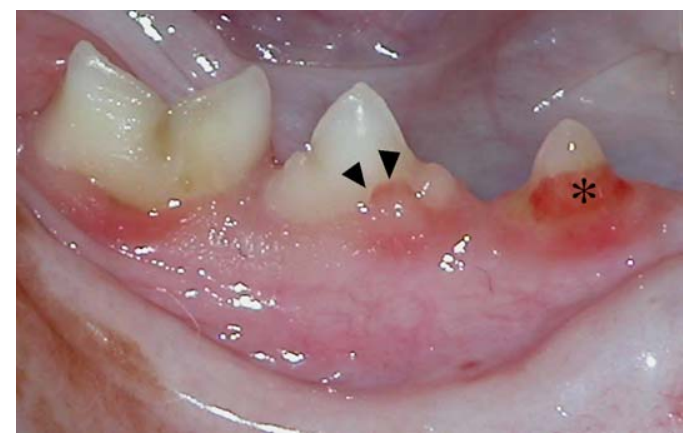

Fig. 1. Classic "neck lesions" at the right lower third (*) and fourth premolar teeth (arrowheads). 


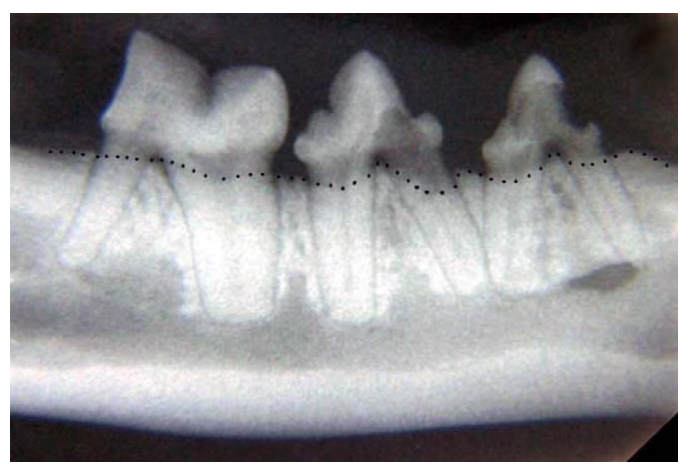

Fig. 2. Radiograph of teeth in Fig. 1; note that inflammatory root resorption is associated with adjacent alveolar bone resorption (dotted line outlining the alveolar margin).

and continues until the roots are completely resorbed or the crown breaks off, leaving root remnants behind [10].

Most previous research focused on FORL emerging at the gingival margin. The commonly observed fusion of roots of feline teeth with alveolar bone (dentoalveolar ankylosis) has not received the same investigative attention. It has previously been reported that the periodontal space is quite narrow in mandibular premolars and molars of adult cats [24]. In a recent histologic study, clinically and radiographically healthy teeth from cats with FORL on other teeth were evaluated. These apparently "healthy" teeth showed hyperemia, edema, and degeneration of the periodontal ligament, with marked fiber disorientation, increased osteoid formation along alveolar bone surfaces (hyperosteoidosis), gradual narrowing of the periodontal space, and areas of ankylotic fusion between the tooth and alveolar bone (Fig. 3) [25]. These findings demonstrate events that occur before resorption and suggest that the early FORL may be noninflammatory in nature [25]. Ankylosed roots are at risk of being incorporated into the normal process of bone remodeling, and the tooth substance is gradually resorbed and replaced by bone (replacement resorption) (Fig. 4) [10]. Ankylosed roots and those with replacement resorption have been categorized radiographically as type II root lesions [18].

\section{Peculiarities of feline permanent teeth}

It has previously been suggested that there is a need for further microscopic research to differentiate histopathologic findings of FORL from normal anatomy of feline teeth [26]. Several peculiarities can be noted in permanent teeth of cats that could represent separate pathologic entities or be associated with FORL.

Cementum is an avascular bone-like tissue covering the roots of mammalian teeth. It normally covers the cervical root surface as a thin 

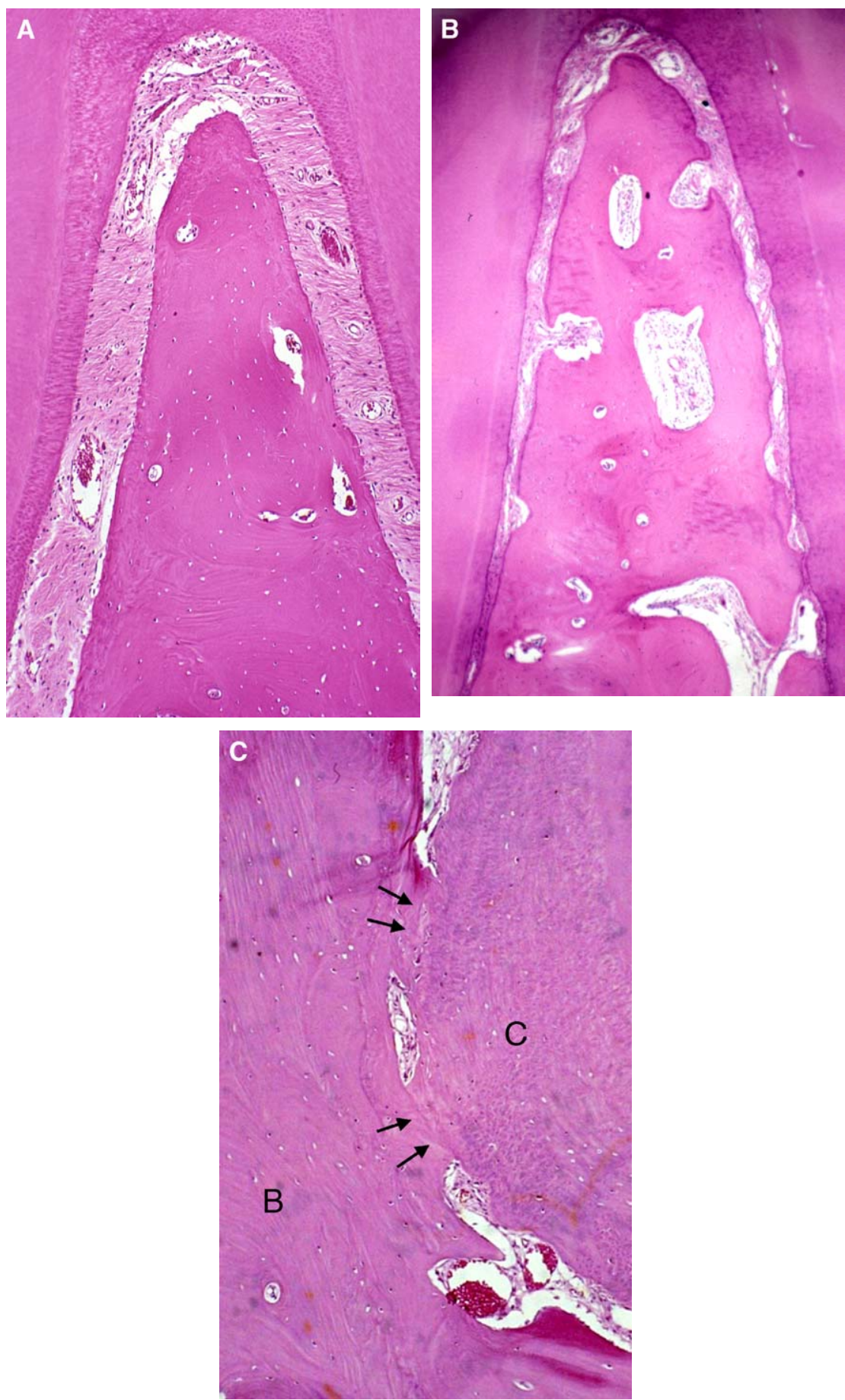


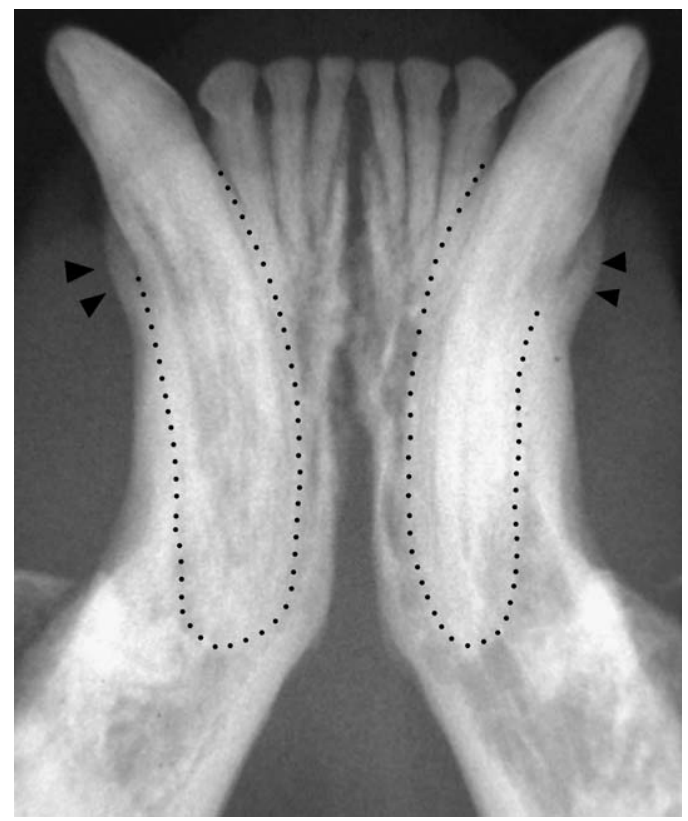

Fig. 4. Radiograph of dentoalveolar ankylosis and root replacement resorption of mandibular canine teeth (dotted line outlining original root contour); also note the bulbous enlargement of crestal alveolar bone (arrowheads).

layer that gradually becomes wider apically. Two types of cementum (acellular and cellular) are usually recognized, which can be further subdivided depending on the presence and origin of collagen fibers (afibrillar, intrinsic, or extrinsic). Cementum formation beyond physiologic deposition is called hypercementosis and can commonly be observed in teeth of cats with FORL [12]. In one study, hypercementosis was demonstrated in all investigated feline teeth [14]. Excessive amounts of cellular cementum are deposited particularly at apical and midroot surfaces, sometimes causing bulbous root apices (Fig. 5), whereas an abnormal thickening of acellular cementum can be found on cervical root surfaces (Fig. 6) [25]. In other species, hypercementosis has been observed in unerupted, hypofunctional, and extruding teeth without opposing antagonists [27-30] and in certain conditions, such as hyperthyroidism [31], hyperpituitarism [32-34], Paget's

Fig. 3. Histopathologic pictures of a feline premolar tooth with a normal furcation area $(A)$ and a premolar tooth of a cat with feline odontoclastic resorptive lesions on other teeth showing degeneration of the periodontal ligament, narrowing of the periodontal space, and dentoalveolar ankylosis $(B)$. Close-up of apical area of tooth root showing periodontal ligament degeneration and two areas of ankylotic fusion (arrows) between cementum $(C)$ and alveolar bone $(B)$. 


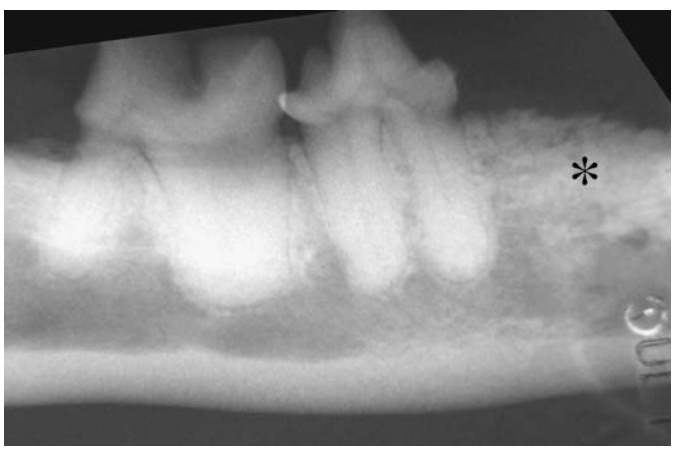

Fig. 5. Radiograph showing bulbous root apices of the right lower fourth premolar and first molar in a cat; note the missing third premolar tooth $(*)$.

disease [35-37], and vitamin A deficiency [38,39]. It has also been demonstrated that occlusal trauma does not lead to hypercementosis [40,41].

Some cats seem to exhibit abnormal extrusion of teeth, referred to as supereruption [10]. Supereruption is most commonly observed in maxillary
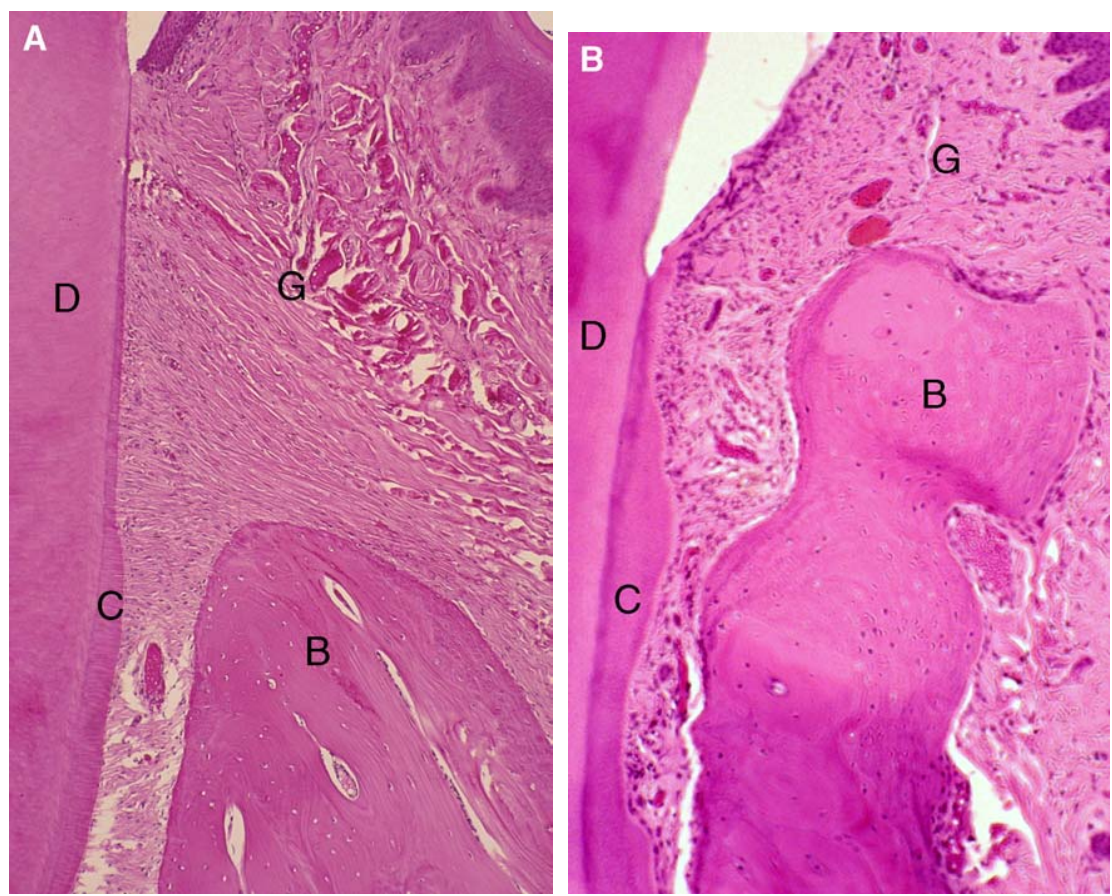

Fig. 6. Histopathologic pictures of a premolar in a cat with thin cervical cementum and normal biologic width $(A)$ and a premolar of a cat with feline odontoclastic resorptive lesions on other teeth showing cervical hypercementosis, bulbous enlargement of crestal alveolar bone, and loss of biologic width $(B)$. B, alveolar bone; C, cementum; D, dentin; $\mathrm{G}$, gingival connective tissue. 
canine teeth, leading to exposure of the root surface (Fig. 7). Normally, active eruption of brachyodont teeth does not cease when they meet their opposing teeth but continues throughout life; ideally, the rate of eruption keeps pace with tooth wear, preserving the vertical dimension of the dentition [42]. It has been speculated that supereruption in cats may be the result of hypercementosis [43] or increased osteoblastic activity of periapical alveolar bone [44]. Another peculiarity found in cats is a distinct thickening of bone along the alveolar margin or the surfaces of the alveolar plates, alone or in combination with supereruption. This alveolar bone expansion is commonly seen in maxillary canine teeth but occurs with less intensity around other teeth as well (Fig. 8) [10]. In human beings, a similar condition is called "peripheral buttressing" and is believed to be a result of the body's attempt to compensate for lost bone during the reparative process associated with trauma from occlusion. The condition may present as shelf-like thickening of the alveolar margin, referred to as "lipping", or as a pronounced bulge in the contour of the alveolar bone [45].

Unusual dentin formation has been described in feline teeth. In one study, osteodentin could be demonstrated in most feline premolars and molars, particularly in furcation areas of root dentin close to the root canal [13]. In osteodentin, cellular inclusions (remnants of odontoblasts) can be found between randomly running dentinal tubules. FORL were observed in areas of the tooth in which osteodentin was most typically found [13]. Vasodentin was found in 3 of 10 control teeth and in 6 of 49 teeth with FORL and was most often observed in the outer third of circumpulpal dentin [46]. In vasodentin, dentinal tubules run randomly, with penetration of canals that may contain vascular-like tissue. Another study found vasodentin almost equally in teeth with or without FORL, although the
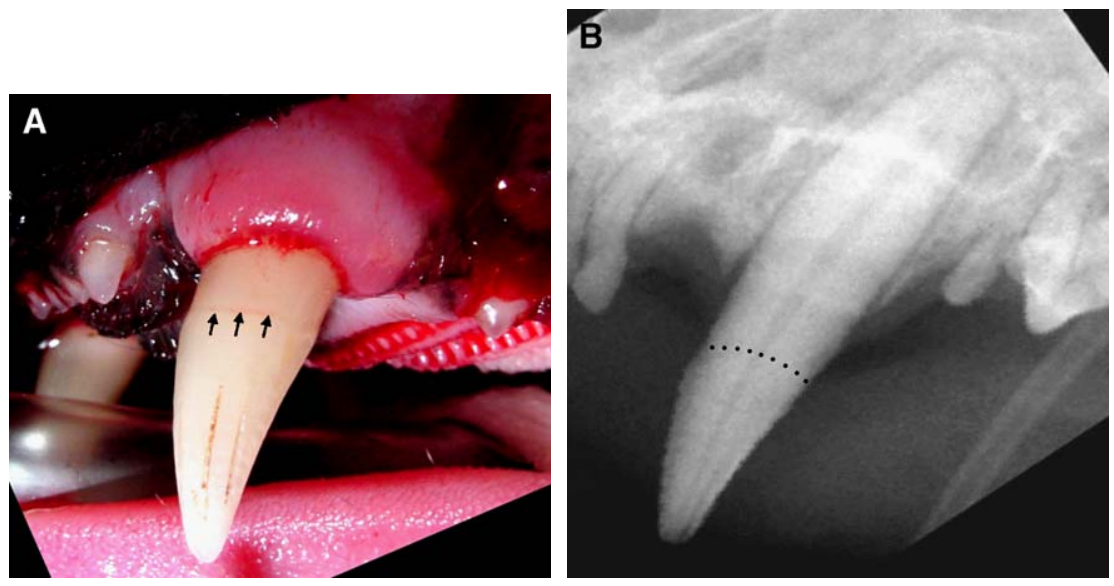

Fig. 7. Clinical picture $(A)$ and radiograph $(B)$ of the left upper canine tooth showing supereruption (arrows and dotted line outlining the cementoenamel junction). 

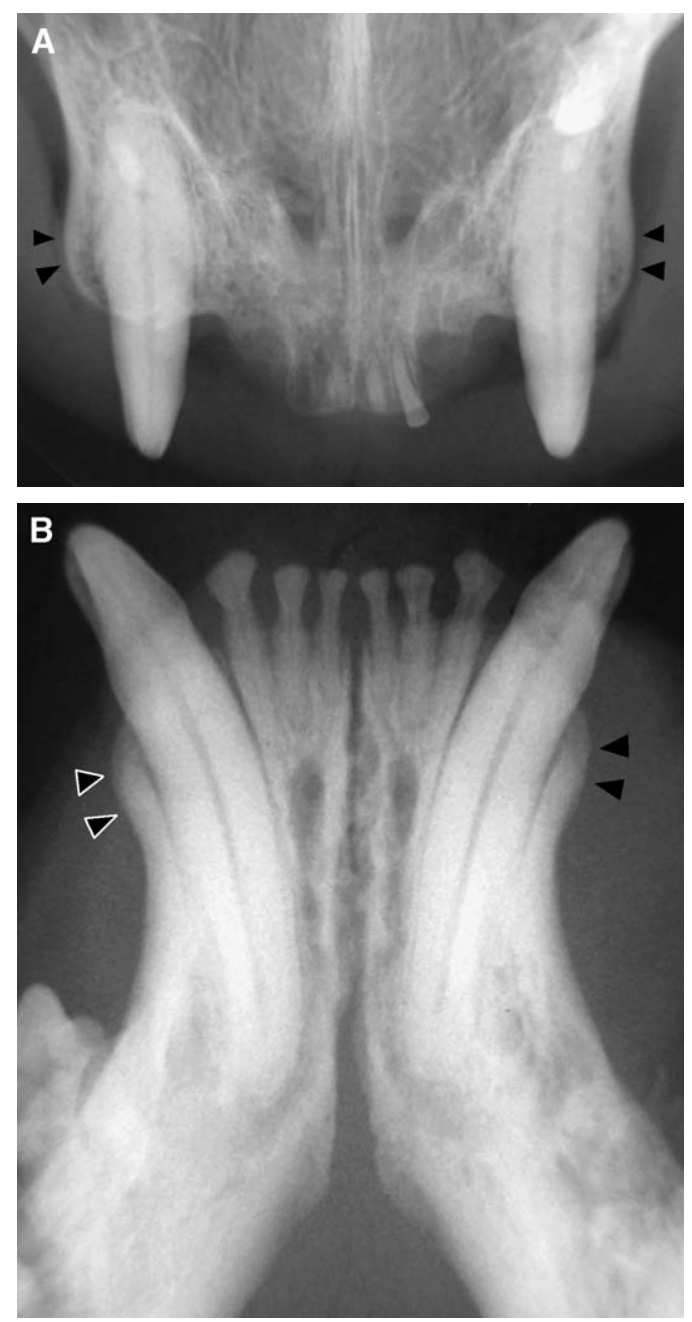

Fig. 8. Radiographs of alveolar bone expansion (arrowheads) of upper $(A)$ and lower canine teeth $(B)$ in cats with missing teeth and feline odontoclastic resorptive lesions on other teeth.

locations of vasodentin and FORL differed [13]. Furcation canals connecting the pulp chamber and the periodontal ligament were found in deciduous premolar teeth in kittens as well as in teeth of adult cats [47,48]. After experimental pulp injury, changes in the periodontal ligament at the opening of the furcation canal and resorption of dental tissues and alveolar bone in the furcation area took place [48]. In a more recent study, patent furcation canals were found in $27 \%$ of permanent carnassial teeth in adult cats [49].

Irregularities in dentin formation are generally considered to be evidence of deficient mineralization during dentinogenesis [50]. The inclusion of 
odontoblasts or pulp tissue into dentin may also be attributable to times of rapid mineralization of newly formed dentin matrix, however. This view is supported by the observation that the layer of predentin appeared extremely thin or was not present in teeth of cats with FORL [51].

\section{Increased vitamin D activity in cats with feline odontoclastic resorptive lesions}

Although FORL may have occurred more than 800 years ago [52], retrospective studies of zoologic collections of feline skulls showed a low prevalence of FORL before the 1960s [53,54]. It was suggested that the increased prevalence of FORL might be associated with aspects of domestication, such as altered feeding practices, vaccination, and neutering programs [10].

Unlike bone that undergoes resorption and apposition as part of a continual remodeling process, the roots of permanent teeth are normally not resorbed because of resorption-inhibiting characteristics of unmineralized layers on external and internal root surfaces (eg, periodontal ligament, cementoblasts and cementoid, odontoblasts and predentin) [10,17]. Odontoclasts may be attracted only to, or can attach only to, mineralized tissue. It has been postulated that removal or mineralization of the organic matrix of the root covering would make it possible for odontoclasts to recognize the mineral component $[10,17]$.

Measurement of biochemical markers of bone turnover, bone alkaline phosphatase (BAP) and deoxypyridinoline (DPD), did not show significant differences between cats with and without FORL [55]. It has recently been demonstrated that cats with FORL expressed a significantly higher mean serum concentration of 25OHD compared with cats without FORL, however [5]. Furthermore, the mean serum concentrations of blood urea nitrogen and phosphorus were significantly higher and the mean urine specific gravity and mean calcium-phosphorus ratio were significantly lower in cats with FORL compared with cats without FORL [5]. Although the mean values of renal parameters remained within physiologic range, the results suggest the possibility of gradual impairment of renal function in cats with FORL. Using a human radioimmunoassay not yet validated for use in cats, calcitonin was significantly more often detected in blood sera of cats with FORL, which may be an expression of protective secretion during times of transient mild hypercalcemia [5]. It was also demonstrated that cats with FORL vomited significantly more often than cats without FORL $[5,56]$.

The diet represents the only source of vitamin D in cats because they are unable to produce vitamin D in the skin [57]. Based on feeding studies in the 1950s, the National Research Council proposed a minimum vitamin D requirement for growing kittens of $500 \mathrm{IU} / \mathrm{kg}$ of dietary dry matter [58]. Later studies demonstrated that kittens given a diet with vitamin $\mathrm{D}_{3}$ per kilogram of dry matter at a rate of 250 or 125 IU did not show clinical signs 
Table 1

Changes in dental and periodontal tissues of experimental animals receiving excess vitamin D or vitamin D metabolites

\begin{tabular}{|c|c|c|c|c|c|c|c|}
\hline $\begin{array}{l}\text { Reference } \\
\text { no. }\end{array}$ & Species & $\begin{array}{l}\text { Age/weight } \\
\text { at start of } \\
\text { experiment }\end{array}$ & $\begin{array}{l}\text { Type of } \\
\text { vitamin D }\end{array}$ & Dose & $\begin{array}{l}\text { Route of } \\
\text { administration }\end{array}$ & $\begin{array}{l}\text { Additional } \\
\text { methods }\end{array}$ & $\begin{array}{l}\text { Diagnostic } \\
\text { tools }\end{array}$ \\
\hline [103] & Rats & $127-182 \mathrm{~g}$ & Vit D (nfd) & $\begin{array}{l}307,000-1,860,000 \\
\text { IU (once); killed } \\
\text { after } 48 \mathrm{~h}\end{array}$ & $\mathrm{SC}$ & $\mathrm{n} / \mathrm{a}$ & $\mathrm{H}$ \\
\hline
\end{tabular}

\begin{tabular}{|c|c|c|c|c|c|c|c|}
\hline [108] & Dogs & $39 \mathrm{~d}$ & $\begin{array}{l}\text { irrad D2 } \\
\text { or D3 }\end{array}$ & $\begin{array}{l}10,000 \mathrm{IU} / \mathrm{kg} \mathrm{BW} \\
\times 9.5 \mathrm{mo}\end{array}$ & Food & $\begin{array}{l}\text { Some dogs } \\
\text { also given } \\
\text { excess vit } \mathrm{A}\end{array}$ & $\mathrm{R}+\mathrm{H}$ \\
\hline$[109,119]$ & Dogs & 29 or $34 \mathrm{~d}$ & irrad D2 & $\begin{array}{l}450,000 \mathrm{IU} \text { (once); } \\
\text { killed at } 2.5,4, \text { or } \\
9 \text { mo of age }\end{array}$ & $\mathrm{PO}$ & $\mathrm{n} / \mathrm{a}$ & $\mathrm{R}+\mathrm{H}$ \\
\hline$[110,114]$ & Dogs & $2 \mathrm{mo}$ & $\mathrm{D} 2$ or $\mathrm{D} 3$ & $\begin{array}{l}10,000 \mathrm{IU} / \mathrm{kg} \mathrm{BW} / \mathrm{d} \\
\times 6 \mathrm{mo} \\
\text { (intermittently) } \\
\text { (total } 1,270,000 \\
\text { and } 1,450,000 \mathrm{IU} \text { ); } \\
\text { killed after } \\
\text { additional } 5 \text { mo of } \\
\text { "recovery period" }\end{array}$ & Food & $\mathrm{n} / \mathrm{a}$ & $\mathrm{R}+\mathrm{H}$ \\
\hline [105] & Rats & $21 \mathrm{~d}(\sim 100 \mathrm{~g})$ & D2 & $\begin{array}{l}500,000 \mathrm{IU} \text { (once); } \\
\text { killed after } 6 \mathrm{~d}\end{array}$ & $\mathrm{P}$ & $\mathrm{n} / \mathrm{a}$ & $\begin{array}{l}\mathrm{R}+\mathrm{H} \\
(\mathrm{I}+\mathrm{M})\end{array}$ \\
\hline [97] & Rats & $40-50 \mathrm{~g}$ & D2 & $\begin{array}{l}100,000 \mathrm{IU} \text { on } 1 \mathrm{st}, \\
4 \mathrm{th}, 7 \mathrm{th}, 10 \mathrm{th} \text {, and } \\
14 \mathrm{th} \mathrm{d} \text {; killed on } 15 \mathrm{th} \mathrm{d}\end{array}$ & IP & $\begin{array}{l}\text { Some rats } \\
\text { also given a } \\
\text { collagen- } \\
\text { damaging } \\
\text { lathyrogen }\end{array}$ & H (M) \\
\hline [121] & Rats & $50-150 \mathrm{~g}$ & D2 & $\begin{array}{l}50,000-200,000 \mathrm{IU} \\
\times 2-4 / \mathrm{wk} ; \text { sacrifice } \\
\text { after } 1-12 \mathrm{wk}\end{array}$ & $\mathrm{PO}$ & $\mathrm{n} / \mathrm{a}$ & $\begin{array}{l}\mathrm{H} \\
(\mathrm{LM}+\mathrm{EM})\end{array}$ \\
\hline [111] & Rats & $154 \mathrm{~g}$ & D2 & $\begin{array}{l}1.25 \mathrm{mio} \mathrm{IU} / \mathrm{kg} \text { of diet } \\
\times 6 \mathrm{wk}\end{array}$ & Food & $\mathrm{n} / \mathrm{a}$ & H (M) \\
\hline [112] & Hamsters & $4 \mathrm{mo}$ & D2 & $\begin{array}{l}5,000 \mathrm{IU} \text { twice } / \mathrm{wk} \\
\times 8 \mathrm{wk}\end{array}$ & IP & $\mathrm{n} / \mathrm{a}$ & H (M) \\
\hline [102] & Pigs & $5 \mathrm{~d}$ & D3 & $\begin{array}{l}45,000-162,000 \mathrm{IU} / \mathrm{d} \\
\times 17-48 \mathrm{~d}\end{array}$ & $\mathrm{PO}$ & $\mathrm{n} / \mathrm{a}$ & $\mathrm{H}$ \\
\hline
\end{tabular}




\begin{tabular}{|c|c|c|c|c|c|}
\hline Pulp/dentin & Cementum & $\begin{array}{l}\text { Periodontal } \\
\text { ligament }\end{array}$ & $\begin{array}{l}\text { Gingival } \\
\text { connective } \\
\text { tissue }\end{array}$ & $\begin{array}{l}\text { Alveolar } \\
\text { bone }\end{array}$ & Comments \\
\hline $\begin{array}{l}\text { Calciotraumatic } \\
\text { line on inner edge } \\
\text { of dentin, } \\
\text { followed by } \\
\text { hypomineralized } \\
\text { layer, wide } \\
\text { hypermineralized } \\
\text { zone, and } \downarrow \text { width } \\
\text { of predentin }\end{array}$ & $\mathrm{n} / \mathrm{r}$ & $\mathrm{n} / \mathrm{r}$ & $\mathrm{n} / \mathrm{r}$ & $\mathrm{n} / \mathrm{r}$ & $\begin{array}{l}\text { Formation of } \\
\text { dentin proceeded } \\
\text { at same rate as } \\
\text { that of control } \\
\text { rats but MIN } \\
\text { was accelerated }\end{array}$ \\
\hline $\begin{array}{l}\text { DEG; pulp stones } \\
\text { in permanent } \\
\mathrm{C}+\mathrm{M}\end{array}$ & $\mathrm{HC}$ & MIN; ANK & MIN & $\begin{array}{l}\text { Initial OP, } \\
\text { followed by } \\
\text { OS with } \downarrow \text { lumen } \\
\text { of ES in younger } \\
\text { dogs; less OS } \\
\text { in older dogs }\end{array}$ & $\begin{array}{l}\downarrow \text { changes in dogs } \\
\text { given vit D from } \\
\text { tuna or halibut } \\
\text { liver oil than } \\
\text { irrad D2; } \\
\downarrow \text { changes in dogs } \\
\text { given excess vit A }\end{array}$ \\
\hline DEG; MIN & $\mathrm{HC}$; resorption & MIN & $\mathrm{n} / \mathrm{r}$ & $\mathrm{OP}$ & $\mathrm{n} / \mathrm{a}$ \\
\hline Pulp stones & $\mathrm{HC}$ & $\begin{array}{l}\text { Development of } \\
\text { granulation tissue } \\
\text { in furcation and } \\
\text { interdental areas; } \\
\text { MIN; ANK }\end{array}$ & MIN & $\begin{array}{l}\text { Increased } \\
\text { vascularity; } \\
\text { granulation } \\
\text { tissue formation; } \\
\uparrow \text { periodontitis } \\
\text { in dog given D3 }\end{array}$ & $\begin{array}{l}\text { OP was } \\
\text { predominant }\end{array}$ \\
\hline $\begin{array}{l}\text { Hemorrhage, } \\
\text { odontoblast } \\
\text { DEG, accelerated } \\
\text { dentin formation, } \\
\text { MIN in M }\end{array}$ & $\mathrm{n} / \mathrm{r}$ & $\mathrm{n} / \mathrm{r}$ & $\mathrm{n} / \mathrm{r}$ & $\mathrm{n} / \mathrm{r}$ & $\mathrm{n} / \mathrm{a}$ \\
\hline $\mathrm{n} / \mathrm{r}$ & $\mathrm{n} / \mathrm{r}$ & MIN & MIN & $\mathrm{n} / \mathrm{r}$ & $\begin{array}{l}\uparrow \text { changes in rats } \\
\text { given the } \\
\text { lathyrogen }\end{array}$ \\
\hline $\mathrm{n} / \mathrm{r}$ & $\begin{array}{l}\text { Intracellular } \\
\text { MIN of } \\
\text { cementoblast-like } \\
\text { cells; HC }\end{array}$ & $\begin{array}{l}\text { DEG; MIN of } \\
\text { fibers close to } \\
\text { cemental surface } \\
\text { ("sunburst" } \\
\text { pattern) }\end{array}$ & MIN & $\begin{array}{l}\text { OP followed, } \\
\text { by } \mathrm{HO} \text { and } \mathrm{OS} \text {; } \\
\text { alveolar crest } \\
\text { raised to CEJ }\end{array}$ & $\mathrm{n} / \mathrm{a}$ \\
\hline $\mathrm{n} / \mathrm{r}$ & $\mathrm{HC}$ & $\begin{array}{l}\text { FD; } \downarrow \text { PS; } \\
\text { MIN; ANK }\end{array}$ & $\begin{array}{l}\text { MIN with } \\
\text { 'sunburst' } \\
\text { pattern near } \\
\text { transeptal } \\
\text { fibers }\end{array}$ & $\begin{array}{l}\text { OP followed, } \\
\text { by HO and OS; } \\
\text { alveolar crest } \\
\text { raised to CEJ; } \\
\text { marrow spaces } \\
\text { filled with young } \\
\text { connective tissue }\end{array}$ & $\mathrm{n} / \mathrm{a}$ \\
\hline $\mathrm{n} / \mathrm{r}$ & $\begin{array}{l}\text { Cemental } \\
\text { spurs }\end{array}$ & $\begin{array}{l}\downarrow \mathrm{PS} ; \mathrm{MIN} ; \\
\text { ANK }\end{array}$ & $\mathrm{n} / \mathrm{r}$ & $\begin{array}{l}\text { Thinning of } \\
\text { cortical bone } \\
\text { and endosteal } \\
\text { resorption, } \\
\text { followed by HO }\end{array}$ & $\mathrm{n} / \mathrm{a}$ \\
\hline $\begin{array}{l}\text { DEG and } \\
\text { hyperemia; MIN; } \\
\text { osteodentin } \\
\text { formation }\end{array}$ & $\begin{array}{l}\text { Resorption of } \\
\text { cementum and } \\
\text { dentin with } \\
\text { pulp exposure }\end{array}$ & $\begin{array}{l}\text { Hyperemia; } \\
\text { MIN; ANK }\end{array}$ & $\mathrm{n} / \mathrm{r}$ & $\begin{array}{l}\text { OP, followed } \\
\text { by } \mathrm{HO}\end{array}$ & $\mathrm{n} / \mathrm{a}$ \\
\hline
\end{tabular}


Table 1 (continued)

\begin{tabular}{|c|c|c|c|c|c|c|c|}
\hline $\begin{array}{l}\text { Reference } \\
\text { no. }\end{array}$ & Species & $\begin{array}{l}\text { Age/weight } \\
\text { at start of } \\
\text { experiment }\end{array}$ & $\begin{array}{l}\text { Type of } \\
\text { vitamin D }\end{array}$ & Dose & $\begin{array}{l}\text { Route of } \\
\text { administration }\end{array}$ & $\begin{array}{l}\text { Additional } \\
\text { methods }\end{array}$ & $\begin{array}{l}\text { Diagnostic } \\
\text { tools }\end{array}$ \\
\hline$[101]$ & Rabbits & $15 \mathrm{~d}(\sim 150 \mathrm{~g})$ & D3 & $\begin{array}{l}600,000 \mathrm{IU} / \mathrm{kg} \mathrm{BW} \\
\text { once } / \mathrm{wk} \times 4 \mathrm{wk} \text {; } \\
\text { killed } 30,45, \text { or } \\
60 \mathrm{~d} \text { after initial } \\
\text { injection }\end{array}$ & IM & $\mathrm{n} / \mathrm{a}$ & $\mathrm{R}+\mathrm{H}$ \\
\hline [106] & Rats & $\mathrm{n} / \mathrm{r}$ & D3 & $\begin{array}{l}10,000 \mathrm{IU} / \mathrm{d} \\
\times 1-4 \mathrm{wk}\end{array}$ & TGT & $\mathrm{n} / \mathrm{a}$ & $\mathrm{H}(\mathrm{I}+\mathrm{M})$ \\
\hline [107] & Rats & $\begin{array}{l}8 \text { or } 12 \text { wk } \\
(35-271 \mathrm{~g})\end{array}$ & D3 & $\begin{array}{l}200,000 \mathrm{IU} / \mathrm{d} \\
(\text { on } 6 \mathrm{~d} / \mathrm{wk} \text { ) } \\
\times \text { up to } 2 \mathrm{mo}\end{array}$ & TGT & $\mathrm{n} / \mathrm{a}$ & $\mathrm{H}(\mathrm{I}+\mathrm{M})$ \\
\hline [122] & Rats & $100 \mathrm{~g}$ & DHT & $50 \mu \mathrm{g} / \mathrm{d} \times 17 \mathrm{~d}$ & TGT & $\mathrm{n} / \mathrm{a}$ & H (M) \\
\hline [123] & Rats & $140-150 \mathrm{~g}$ & DHT & $\begin{array}{l}50 \mu \mathrm{g} / \mathrm{d} \times 31 \\
\text { or } 62 \mathrm{~d}\end{array}$ & TGT & $\begin{array}{l}\text { Some rats } \\
\text { also given } \\
\text { FD }\end{array}$ & H (M) \\
\hline [120] & Rats & $\sim 220 \mathrm{~g}$ & DHT & $50 \mu \mathrm{g} / \mathrm{d} \times 50 \mathrm{~d}$ & TGT & $\mathrm{n} / \mathrm{a}$ & $\mathrm{H}$ \\
\hline [91] & Rats & $215 \mathrm{~g}$ & DHT & $50 \mu \mathrm{g} / \mathrm{d} \times 50 \mathrm{~d}$ & $\mathrm{PO}$ & $\begin{array}{l}\text { Some rats } \\
\text { also given } \\
\text { FD }\end{array}$ & $\mathrm{H}(\mathrm{M})$ \\
\hline [98] & Rats & $200 \mathrm{~g}$ & DHT & $50 \mu \mathrm{g} / \mathrm{d} \times 7-50 \mathrm{~d}$ & TGT & $\begin{array}{l}\text { Some rats } \\
\text { also given } \\
\text { FD }\end{array}$ & $\mathrm{H}$ \\
\hline [95] & Rats & $\sim 100 \mathrm{~g}$ & DHT & $50 \mu \mathrm{g} / \mathrm{d} \times 40 \mathrm{~d}$ & TGT & $\begin{array}{l}\text { Some rats } \\
\text { also given } \\
\text { TS }\end{array}$ & $\mathrm{H}(\mathrm{M})$ \\
\hline
\end{tabular}




\begin{tabular}{|c|c|c|c|c|c|}
\hline Pulp/dentin & Cementum & $\begin{array}{l}\text { Periodontal } \\
\text { ligament }\end{array}$ & $\begin{array}{l}\text { Gingival } \\
\text { connective } \\
\text { tissue }\end{array}$ & $\begin{array}{l}\text { Alveolar } \\
\text { bone }\end{array}$ & Comments \\
\hline $\mathrm{n} / \mathrm{r}$ & $\mathrm{n} / \mathrm{r}$ & FD; MIN & $\mathrm{n} / \mathrm{r}$ & $\begin{array}{l}\text { OP, followed by } \\
\mathrm{HO} \text { and OS }\end{array}$ & $\mathrm{n} / \mathrm{a}$ \\
\hline $\begin{array}{l}\text { Pulp stones } \\
\text { in I }\end{array}$ & $\mathrm{HC}$ & $\begin{array}{l}\text { MIN; } \\
\text { ANK in M }\end{array}$ & $\mathrm{n} / \mathrm{r}$ & $\mathrm{HO}$ and $\mathrm{OS}$ & $\mathrm{n} / \mathrm{a}$ \\
\hline $\begin{array}{l}\downarrow \text { width of } \\
\text { predentin; } \\
\text { DEG of } \\
\text { odontoblasts; } \\
\text { pulp stones } \\
\text { (primarily } \\
\text { in I of young } \\
\text { and older rats) }\end{array}$ & $\begin{array}{l}\mathrm{HC} \text { (most intense } \\
\text { in apical areas of } \\
\text { young rats); } \\
\text { resorption of } \\
\text { cementum and } \\
\text { dentin in nearly } \\
\text { all } \mathrm{M} \text { of rat fed } \\
\text { longest with D3 }\end{array}$ & $\begin{array}{l}\downarrow \mathrm{PS} ; \mathrm{MIN} ; \\
\text { ANK in M }\end{array}$ & $\mathrm{n} / \mathrm{r}$ & $\begin{array}{l}\text { OP, followed by } \\
\text { HO and OS } \\
\text { (predominantly in } \\
\text { young rats); } \\
\downarrow \text { lumen of ES; } \\
\uparrow \text { crestal } \\
\text { alveolar bone } \\
\text { (predominantly } \\
\text { in young rats) }\end{array}$ & $\mathrm{n} / \mathrm{a}$ \\
\hline $\begin{array}{l}\text { Hyperemia, } \\
\text { hemorrhage, } \\
\text { and separation } \\
\text { of odontoblasts }\end{array}$ & $\mathrm{HC}$ & $\begin{array}{l}\text { DEG, edema, } \\
\text { and hemorrhage; } \\
\text { FD; MIN; ANK }\end{array}$ & $\mathrm{n} / \mathrm{r}$ & $\begin{array}{l}\mathrm{HO} ; \downarrow \downarrow \text { lumen of ES; } \\
\text { edema of bone } \\
\text { marrow }\end{array}$ & $\mathrm{n} / \mathrm{a}$ \\
\hline $\begin{array}{l}\text { Edema, } \\
\text { hyperemia, } \\
\text { hemorrhage, } \\
\text { and reticular } \\
\text { atrophy; } \\
\text { pulp stones }\end{array}$ & $\begin{array}{l}\text { HC; "club"-shaped } \\
\text { root apices; } \\
\text { resorption of } \\
\text { cementum and } \\
\text { dentin, particularly } \\
\text { in furcation areas }\end{array}$ & $\begin{array}{l}\text { DEG, edema, and } \\
\text { hemorrhage; FD; } \\
\downarrow \text { PS; MIN; ANK }\end{array}$ & $\mathrm{n} / \mathrm{r}$ & $\begin{array}{l}\mathrm{HO} ; \downarrow \text { lumen of ES; } \\
\text { bulbous } \\
\text { enlargement of } \\
\text { alveolar plates; } \\
\text { edematous } \\
\text { marrow tissue }\end{array}$ & $\begin{array}{l}\downarrow \text { changes in rats } \\
\text { given FD }\end{array}$ \\
\hline $\mathrm{n} / \mathrm{r}$ & $\begin{array}{l}\text { HC; "club"-shaped } \\
\text { root apices; } \\
\text { resorption of } \\
\text { cementum and } \\
\text { dentin with } \\
\text { ingrowth of } \\
\text { connective tissue } \\
\text { cells into resorptive } \\
\text { defects }\end{array}$ & $\begin{array}{l}\mathrm{FD} ; \downarrow \text { PS; MIN; } \\
\text { ANK }\end{array}$ & $\begin{array}{l}\text { MIN with } \\
\text { 'sunburst' } \\
\text { pattern near } \\
\text { transeptal } \\
\text { fibers }\end{array}$ & $\begin{array}{l}\text { Rapid and } \\
\text { progressive } \\
\text { resorption, } \\
\text { followed by } \\
\text { HO and OS }\end{array}$ & $\mathrm{n} / \mathrm{a}$ \\
\hline $\mathrm{n} / \mathrm{r}$ & $\mathrm{HC}$ & DEG; FD; ANK & $\begin{array}{l}\text { MIN with } \\
\text { 'sunburst' } \\
\text { pattern near } \\
\text { transeptal } \\
\text { fibers }\end{array}$ & $\begin{array}{l}\text { HO and OS; } \\
\downarrow \text { lumen of ES; } \\
\text { bulbous } \\
\text { enlargement of } \\
\text { alveolar plates }\end{array}$ & $\begin{array}{l}\downarrow \text { changes in rats } \\
\text { given FD; most } \\
\text { severe changes in } \\
\text { furcation areas }\end{array}$ \\
\hline $\mathrm{n} / \mathrm{r}$ & $\begin{array}{l}\text { HC ("club"-shaped } \\
\text { root apices) }\end{array}$ & $\begin{array}{l}\text { DEG, hyperemia, } \\
\text { and edema; } \\
\downarrow \text { PS; MIN; ANK }\end{array}$ & $\begin{array}{l}\text { MIN with } \\
\text { 'sunburst' } \\
\text { pattern near } \\
\text { transeptal } \\
\text { fibers }\end{array}$ & $\begin{array}{l}\mathrm{HO} \text { and OS; } \\
\text { bulbous } \\
\text { enlargement of } \\
\text { alveolar plates } \\
\text { causing coronal } \\
\text { displacement of } \\
\text { transeptal fibers; } \\
\text { hyperemia and } \\
\text { progressive fibrosis } \\
\text { of bone marrow }\end{array}$ & $\begin{array}{l}\downarrow \text { changes in rats } \\
\text { given FD; most } \\
\text { severe changes in } \\
\text { furcation areas }\end{array}$ \\
\hline $\begin{array}{l}\text { Hemorrhage; } \\
\text { pulp stones }\end{array}$ & $\mathrm{HC}$ & $\begin{array}{l}\text { DEG, hyperemia, } \\
\text { and edema; } \\
\downarrow \text { PS; ANK }\end{array}$ & $\mathrm{n} / \mathrm{r}$ & $\begin{array}{l}\mathrm{HO} ; \downarrow \text { lumen of ES; } \\
\text { fibrosis of bone } \\
\text { marrow; } \\
\text { enlargement of } \\
\text { buccal and lingual } \\
\text { bone at areas of } \\
\text { muscle insertion }\end{array}$ & $\begin{array}{l}\downarrow \text { changes in rats } \\
\text { given TS }\end{array}$ \\
\hline
\end{tabular}


Table 1 (continued)

\begin{tabular}{|c|c|c|c|c|c|c|c|}
\hline $\begin{array}{l}\text { Reference } \\
\text { no. }\end{array}$ & Species & $\begin{array}{l}\text { Age/weight } \\
\text { at start of } \\
\text { experiment }\end{array}$ & $\begin{array}{l}\text { Type of } \\
\text { vitamin D }\end{array}$ & Dose & $\begin{array}{l}\text { Route of } \\
\text { administration }\end{array}$ & $\begin{array}{l}\text { Additional } \\
\text { methods }\end{array}$ & $\begin{array}{l}\text { Diagnost } \\
\text { tools }\end{array}$ \\
\hline [96] & Rats & $\sim 260 \mathrm{~g}$ & DHT & $\begin{array}{l}1 \mathrm{mg} / 100 \mathrm{~g} \mathrm{BW} \\
\text { (once); killed after } \\
10,17 \text { or } 31 \mathrm{~d}\end{array}$ & TGT & $\begin{array}{l}\text { Gingival } \\
\text { wound } \\
\text { created } 3 \mathrm{~d} \\
\text { after DHT } \\
\text { was given }\end{array}$ & H (M) \\
\hline$[125]$ & Rats & $40 \mathrm{~d}$ & DHT & $50 \mu \mathrm{g} / \mathrm{d} \times 50 \mathrm{~d}$ & TGT & $\mathrm{n} / \mathrm{a}$ & H (M) \\
\hline [99] & Rats & $\sim 100 \mathrm{~g}$ & DHT & $50 \mathrm{mg} / \mathrm{d} \times 1-7 w \mathrm{k}$ & TGT & $\begin{array}{l}\text { Some rats } \\
\text { had all L } \\
\text { max M } \\
\text { extracted }\end{array}$ & H (M) \\
\hline [117] & Rats & $100 \mathrm{~g}$ & D2 or DHT & $\begin{array}{l}10,000 \mathrm{IU}(\mathrm{D} 2) / \mathrm{d} \\
\text { or } 50 \mu \mathrm{g}(\mathrm{DHT}) / \mathrm{d} \\
\times 50 \mathrm{~d}\end{array}$ & $\begin{array}{l}\mathrm{SC}(\mathrm{D} 2) \\
\text { or TGT } \\
(\mathrm{DHT})\end{array}$ & $\begin{array}{l}\text { Some rats } \\
\text { also given } \\
\text { TS or ED }\end{array}$ & H (M) \\
\hline [116] & Rats & $100 \mathrm{~g}$ & DHT & $50 \mu \mathrm{g} / \mathrm{d} \times 7-35 \mathrm{~d}$ & TGT & $\mathrm{n} / \mathrm{a}$ & $\mathrm{H}(\mathrm{M})$ \\
\hline [100] & Rats & $180-220 \mathrm{~g}$ & DHT & $\begin{array}{l}50 \mu \mathrm{g} / 100 \mathrm{~g} \mathrm{BW} / \mathrm{d} \\
\times 28 \mathrm{~d}\end{array}$ & TGT & $\begin{array}{l}\text { Traumatic } \\
\text { occlusion } \\
\text { induced } \\
\text { in some rats }\end{array}$ & H (M) \\
\hline [118] & Rats & $5 \mathrm{wk}$ & DHT & $\begin{array}{l}50 \mu \mathrm{g} / 100 \mathrm{~g} \mathrm{BW} / \mathrm{d} \\
\times 28 \mathrm{~d}\end{array}$ & TGT & $\begin{array}{l}\text { Some rats } \\
\text { also given } \\
\text { SF }\end{array}$ & H (M) \\
\hline [124] & Rats & $140 \mathrm{~g}$ & DHT & $\begin{array}{l}50 \mu \mathrm{g} / 100 \mathrm{~g} \mathrm{BW} / \mathrm{d} \\
\times \text { up to } 20 \mathrm{~d}\end{array}$ & TGT & $\mathrm{n} / \mathrm{a}$ & \\
\hline [115] & Rats & $6 \mathrm{wk}$ & DHT & $\begin{array}{l}25 \text { or } 50 \mu \mathrm{g} / \mathrm{d} \\
\times 1-4 \mathrm{wk}\end{array}$ & TGT & $\mathrm{n} / \mathrm{a}$ & $\mathrm{H}(\mathrm{M})$ \\
\hline [104] & Rats & $4 \mathrm{wk}$ & $\begin{array}{l}1,25(\mathrm{OH}) \\
2 \mathrm{D} 3\end{array}$ & $\begin{array}{l}0.075 \mu \mathrm{g} / \mathrm{d} \\
\times 5 \mathrm{wk}\end{array}$ & $\mathrm{SC}$ & $\mathrm{n} / \mathrm{a}$ & $\begin{array}{l}\mathrm{H}+\mathrm{R} \\
(\mathrm{I}+\mathrm{M})\end{array}$ \\
\hline
\end{tabular}

Abbreviations: ANK, ankylosis; BW, body weight; C, canine teeth; CEJ, cementoenamel junction; d, days; D2, vitamin D $;$, D3, vitamin $\mathrm{D}_{3}$; DEG, degeneration; DHT, dihydrotachysterol; ED, estradiol; EM, electron microscopy; FD, ferric dextran; h, hours; H, histology; HC, hypercementosis; HO, hyperosteoidosis; I, incisor teeth; IM, intramuscular junction; IP, intraperitoneal injection; irrad; irradiation; L, left; LM, light microscopy; M, molar teeth; max, maxillary; MIN, mineralization; mio, million; mo, months; nfd, not further defined; n/a, not applicable; n/r, not reported; 1,25(OH)2D3, 1,25-dyhydroxyvitamin $\mathrm{D}_{3}$; OP, osteoporosis; OS, osteosclerosis; P, parenteral; PO, per os; PS, periodontal space; R, radiography; SC, subcutaneous; SF, sodium fluoride; TGT, transoral gastric tube; TS, testosterone; vit, vitamin; wk, weeks. 


\begin{tabular}{|c|c|c|c|c|c|}
\hline Pulp/dentin & Cementum & $\begin{array}{l}\text { Periodontal } \\
\text { ligament }\end{array}$ & $\begin{array}{l}\text { Gingival } \\
\text { connective } \\
\text { tissue }\end{array}$ & $\begin{array}{l}\text { Alveolar } \\
\text { bone }\end{array}$ & Comments \\
\hline $\mathrm{n} / \mathrm{r}$ & $\mathrm{HC}$ & ANK & $\mathrm{n} / \mathrm{r}$ & $\begin{array}{l}\text { HO; new bone } \\
\text { formation at } \\
\text { alveolar crest } \\
\text { below the injury }\end{array}$ & $\mathrm{n} / \mathrm{a}$ \\
\hline $\begin{array}{l}\downarrow \text { number of } \\
\text { pulp cells; } \\
\text { MIN; } \\
\downarrow \text { lumen of } \\
\text { pulp cavity }\end{array}$ & $\mathrm{HC}$ & $\begin{array}{l}\text { DEG and FD; } \\
\downarrow \text { PS; MIN }\end{array}$ & $\mathrm{n} / \mathrm{r}$ & $\begin{array}{l}\text { HO and OS; } \\
\downarrow \text { lumen of ES; } \\
\text { bulbous } \\
\text { enlargement of } \\
\text { alveolar plates }\end{array}$ & $\mathrm{n} / \mathrm{a}$ \\
\hline $\mathrm{n} / \mathrm{r}$ & $\mathrm{HC}$ & $\begin{array}{l}\text { DEG and } \\
\text { FD } ; \downarrow \text { PS }\end{array}$ & $\mathrm{n} / \mathrm{r}$ & $\begin{array}{l}\text { HO and OS; } \\
\downarrow \text { lumen of ES; } \\
\text { fibrosis of bone } \\
\text { marrow; bulbous } \\
\text { enlargement of } \\
\text { alveolar plates }\end{array}$ & $\begin{array}{l}\downarrow \text { changes in male } \\
\text { rats and teeth } \\
\text { without opposing } \\
\text { antagonists }\end{array}$ \\
\hline $\mathrm{n} / \mathrm{r}$ & $\mathrm{HC}$ & $\begin{array}{l}\text { DEG, } \\
\text { hyperemia, } \\
\text { and FD; } \\
\downarrow \text { PS; MIN }\end{array}$ & $\mathrm{n} / \mathrm{r}$ & $\begin{array}{l}\mathrm{HO} ; \downarrow \text { lumen } \\
\text { of ES }\end{array}$ & $\begin{array}{l}\downarrow \text { changes in rats } \\
\text { given D2; when } \\
\text { given DHT, } \\
\uparrow \text { changes in } \\
\text { female rats; } \\
\downarrow \text { changes in rats } \\
\text { given DHT } \\
\text { when also given } \\
\text { sexual hormones }\end{array}$ \\
\hline $\mathrm{n} / \mathrm{r}$ & $\mathrm{HC}$ & $\begin{array}{l}\text { DEG and FD; } \\
\text { MIN; ANK }\end{array}$ & $\mathrm{n} / \mathrm{r}$ & HO & $\mathrm{n} / \mathrm{a}$ \\
\hline $\mathrm{n} / \mathrm{r}$ & $\mathrm{HC}$ & $\mathrm{FD} ; \downarrow \mathrm{PS}$ & $\mathrm{n} / \mathrm{r}$ & $\mathrm{HO}$ & $\begin{array}{l}\uparrow \text { changes in rats } \\
\text { with traumatic } \\
\text { occlusion }\end{array}$ \\
\hline $\mathrm{n} / \mathrm{r}$ & $\mathrm{HC}$ & $\mathrm{FD} ; \downarrow \mathrm{PS}$ & $\mathrm{n} / \mathrm{r}$ & $\mathrm{HO}$ & $\begin{array}{l}\downarrow \text { changes in rats } \\
\text { given FD }\end{array}$ \\
\hline $\mathrm{n} / \mathrm{r}$ & $\mathrm{HC}$ & $\begin{array}{l}\text { DEG and FD; } \\
\downarrow \text { PS; ANK }\end{array}$ & $\mathrm{n} / \mathrm{r}$ & $\mathrm{HO}$ & $\mathrm{n} / \mathrm{a}$ \\
\hline $\mathrm{n} / \mathrm{r}$ & $\mathrm{HC}$ & $\begin{array}{l}\text { DEG; } \downarrow \text { PS; } \\
\text { ANK }\end{array}$ & $\mathrm{n} / \mathrm{r}$ & $\begin{array}{l}\mathrm{HO} ; \downarrow \downarrow \text { lumen } \\
\text { of ES; fibrosis } \\
\text { of bone marrow }\end{array}$ & $\begin{array}{l}\text { Progeria-like } \\
\text { changes }\end{array}$ \\
\hline $\begin{array}{l}\downarrow \text { width of } \\
\text { predentin; } \\
\text { DEG of } \\
\text { odontoblasts } \\
\text { and fibroblasts; } \\
\text { formation of } \\
\text { irregular dentin } \\
\text { and osteodentin }\end{array}$ & $\mathrm{HC}$ & ANK of M & $\mathrm{n} / \mathrm{r}$ & $\mathrm{HO}$ & $\mathrm{n} / \mathrm{a}$ \\
\hline
\end{tabular}



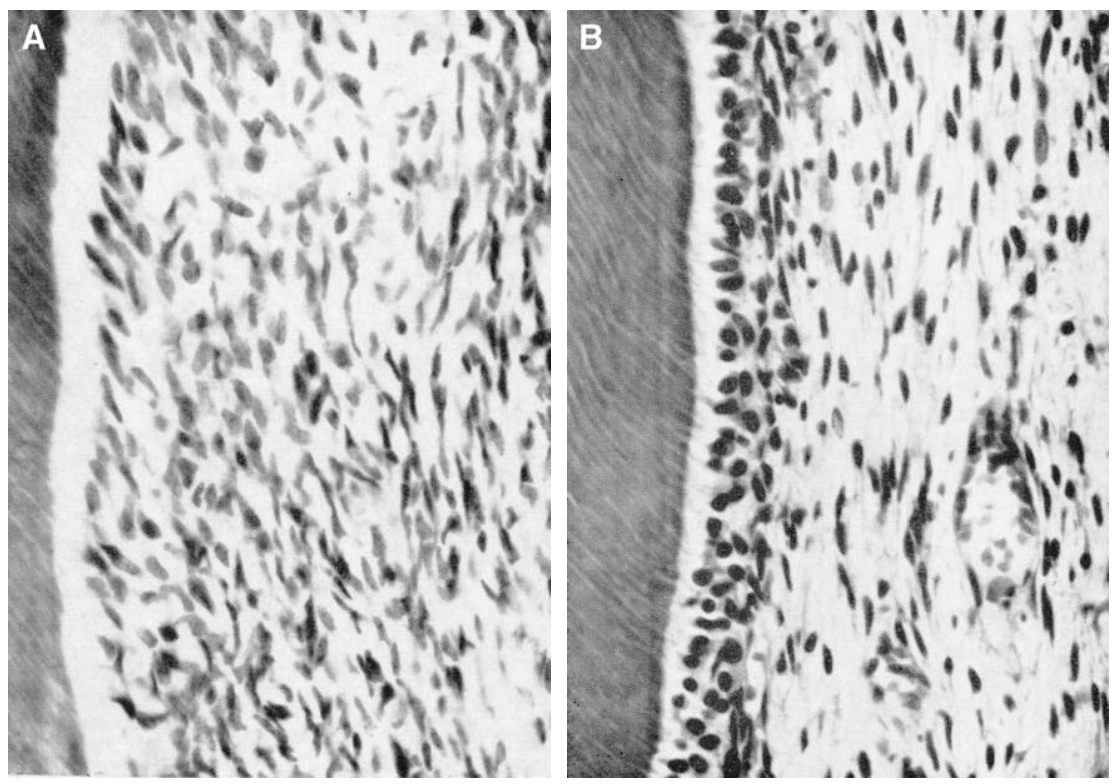

Fig. 9. Histopathologic pictures of pulp from molar teeth of a control rat $(A)$ and pulp from a molar tooth of a rat given dihydrotachysterol showing increased activity of odontoblasts, fluid accumulation in the odontoblastic layer, and reticular atrophy with hyperemia and edema $(B)$. (From Ratcliff PA, Itokazu H. The effect of dihydrotachysterol on the teeth and periodontium. J Periodontol 1964;35:324; with permission.)

of vitamin D deficiency [59,60]. Furthermore, it was found that one third of commercial cat foods contained vitamin $\mathrm{D}_{3}$ in excess of current maximal allowances $(>10,000 \mathrm{IU} / \mathrm{kg}$ of dietary dry matter $)$, and a direct linear relation was demonstrated between 25OHD serum concentrations and dietary intake of vitamin D [61]. Therefore, higher 25OHD serum concentrations in cats with FORL suggest that they had ingested larger amounts of vitamin D or vitamin D metabolites compared with cats without FORL [5]. Three separate incidences of fatal hypervitaminosis D were reported in cats in Japan after consumption of commercial cat foods prepared from fish [62-64]. Clinical, laboratory, and histopathologic findings in these cats included vomiting, hypercalcemia, hyperphosphatemia, azotemia, proteinuria, calciuria, phosphaturia, decreased urine specific gravity, and mineralization of various body tissues, particularly the kidneys and walls of large blood vessels [62]. One may speculate as to whether there is indeed a predisposition to impairment of renal function in cats with FORL, because results of experimental studies on cats fed diets high in vitamin $\mathrm{D}_{3}(15,000-33,840 \mathrm{IU} / \mathrm{kg}$ of dry matter) were contradictory, ranging from no evidence of detrimental effects on feline health to a high prevalence of renal dysfunction and mortality [65]. 

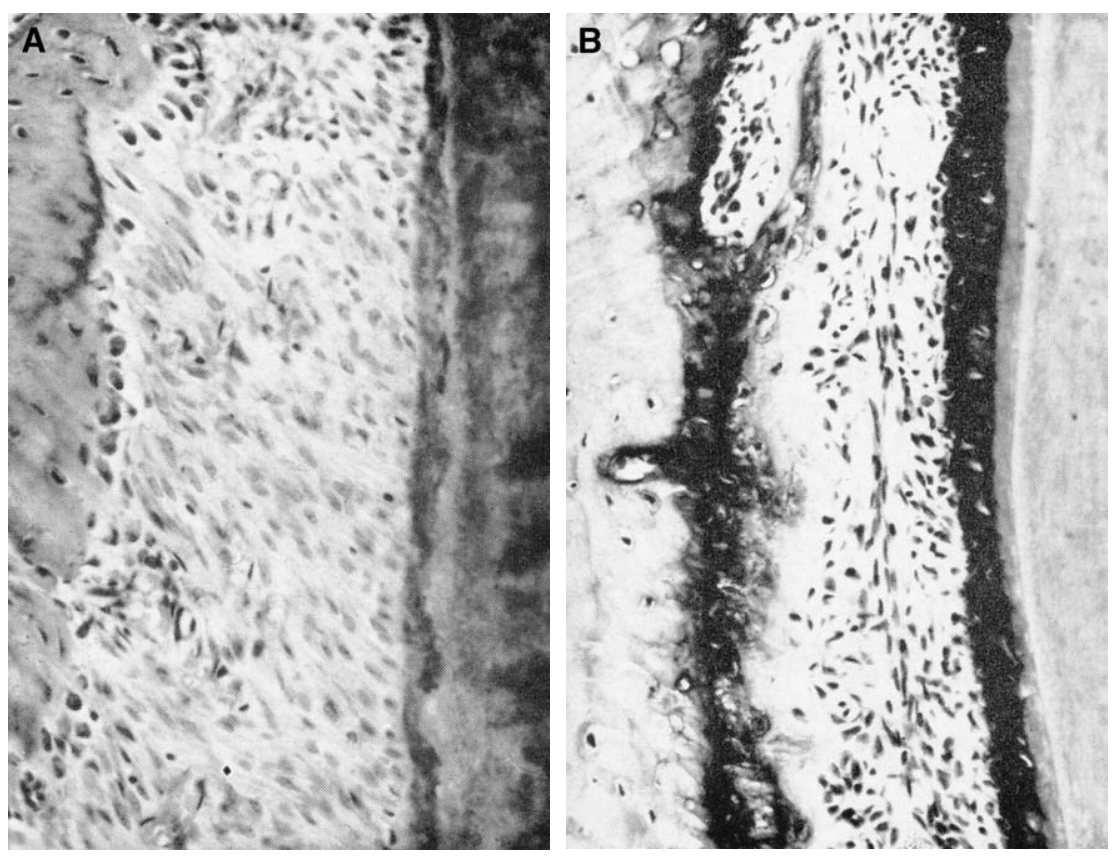

Fig. 10. Histopathologic picture showing periodontal space from molar teeth of a control rat (A) and a rat given dihydrotachysterol showing periodontal ligament fiber disorientation, edema, hyperemia, hypercementosis, hyperosteoidosis with bone spur formation, and narrowing of the periodontal space $(B)$. (From Ratcliff PA, Itokazu H. The effect of dihydrotachysterol on the teeth and periodontium. J Periodontol 1964;35:323; with permission.)

Vitamin D and vitamin D metabolites are important regulators of osteoclastic bone resorption [66]. Serum calcium concentration is maintained within a normal range through the primary action of 1,25-dihydroxyvitamin $\mathrm{D}_{3}\left[1,25(\mathrm{OH})_{2} \mathrm{D}_{3}\right]$, which increases intestinal absorption of dietary calcium and recruits hematopoietic stem cells to become osteoclasts. Osteoclasts, in turn, mobilize calcium stores from bone into the circulation. Osteoclasts do not possess receptors for $1,25(\mathrm{OH})_{2} \mathrm{D}_{3}$, however [66]. Receptors for $1,25(\mathrm{OH})_{2} \mathrm{D}_{3}$ are located on osteoblasts that produce factors stimulating osteoclasts, such as receptor activator of nuclear factor- $\kappa \mathrm{B}$ ligand (RANKL), which plays an important role in osteoclastogenesis [67] and osteoclast activation [68].

\section{Role of local trauma}

The occlusal stress (tooth flexure) theory was created in an attempt to explain noncarious cervical lesions, an overall term for tooth wear (not resorption) at the cervical portion of human teeth [69-71]. Repeated compressive and tensile forces attributable to tooth flexure during 

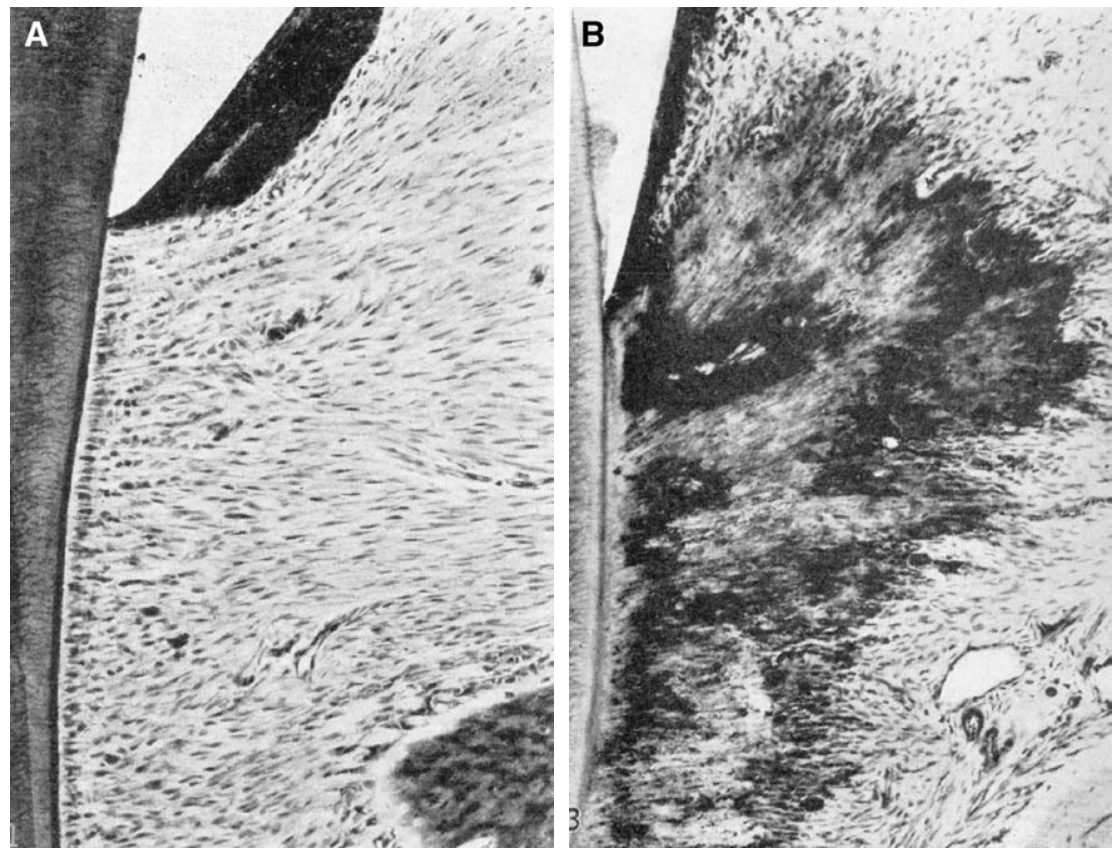

Fig. 11. Histopathologic pictures of cervical portion from teeth of a control dog $(A)$ and a $\operatorname{dog}$ given excessive amounts of vitamin D showing abnormal thickening of cervical cementum $(B)$. (From Becks H. Dangerous effects of vitamin D overdosage on dental and paradental structures. J Am Dent Assoc 1942;29:1960; with permission.)

mastication and malocclusion may disrupt the bonds between enamel rods and between enamel and dentin, resulting in abfraction of enamel, exposure of dentin, and cervical hypersensitivity $[72,73]$. Although FORL are clearly resorptive in nature and develop on any tooth and any root surface (not just on those exposed to occlusal or shearing forces), occlusal stress caused by eating large dry kibbles has been suggested to be associated with FORL $[18,74,75]$. A different approach for a possible role of occlusal stress in the development of FORL is presented in this article.

Surface resorption of cementum and superficial dentin may develop in response to normal masticatory stress [76] and excessive occlusal force [7780]. Apical root resorption has been linked with bruxism in human beings, although the apical defect in that case report could also have resulted from endodontic disease [81]. Traumatic occlusion from maloccluding teeth may cause resorption of roots in rats and people, with the apical area being affected most often [22,82-86]. Root resorption has been demonstrated after experimental intrusion of teeth in people [87] and long-standing occlusal trauma in dogs and monkeys $[88,89]$. Subsequent repairs could eventually result in ankylosis [90]. 

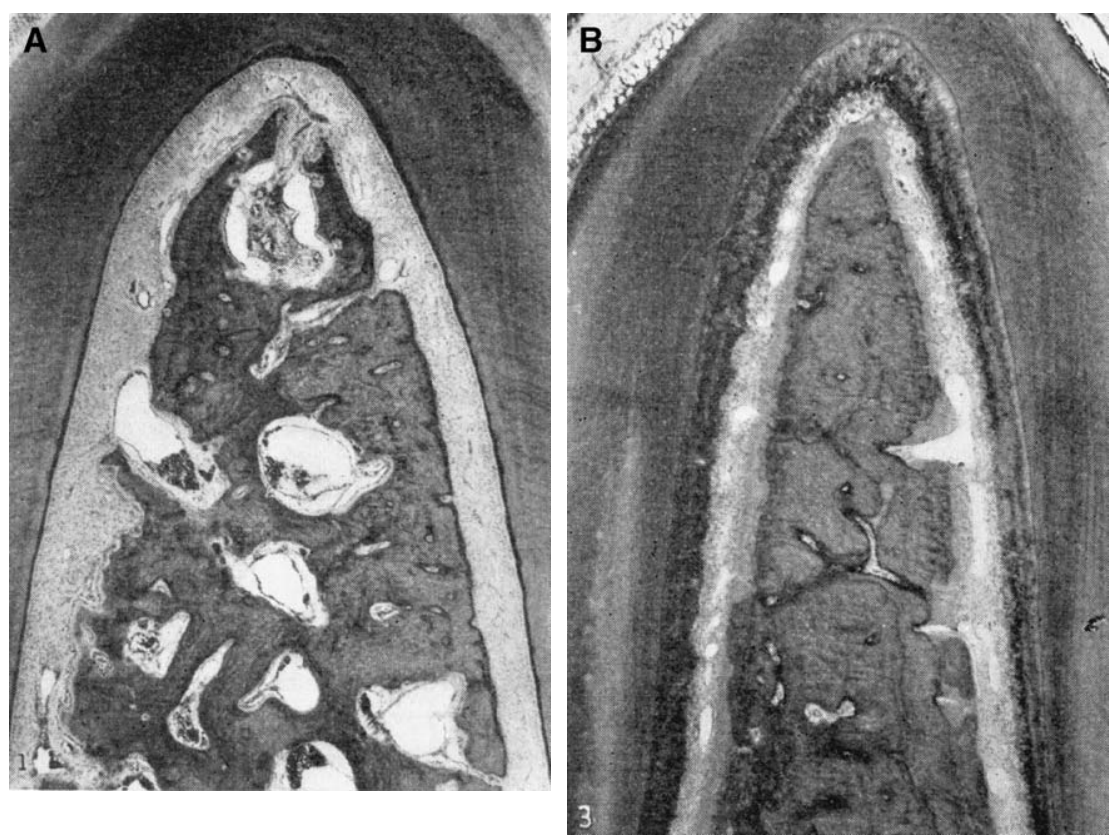

Fig. 12. Histopathologic pictures of furcation area from teeth of a control $\operatorname{dog}(A)$ and a $\operatorname{dog}$ given excessive amounts of vitamin $\mathrm{D}$ showing hypercementosis, hyperosteoidosis, and narrowing of the periodontal space $(B)$. (From Becks H. Dangerous effects of vitamin D overdosage on dental and paradental structures. J Am Dent Assoc 1942;29:1951; with permission.)

Calciphylaxis is a condition of induced local or systemic hypersensitivity in which tissues respond to appropriate challenging agents with precipitous, sometimes evanescent, local mineralization of various tissues and organs [91,92]. Substances that predispose the organism to calciphylaxis are known as sensitizers. Sensitizers are systemically administered agents that promote mineralization of tissues and include vitamin D and vitamin D metabolites, parathyroid hormone, and sodium acetylsulfathiazole among many other calcium salts and phosphates [91]. Agents that precipitate the calciphylaxis phenomenon are known as challengers. Challengers may be direct or indirect. Direct challengers include mechanical trauma and various chemical agents (eg, salts of iron, chromium, aluminum, zinc, manganese, cesium) that cause mineralization at the site of application and may elicit some form of systemic calciphylaxis when administered intravenously or intraperitoneally. Indirect challengers have little or no effect at the site of application and produce diverse systemic syndromes of mineralization and sclerosis [91].

Experiments in dihydrotachysterol (DHT)-sensitized rats indicated that functional stress and topical trauma can produce local calcium deposits in various parts of the body $[91,93,94]$. In rats given DHT, enlargement of 

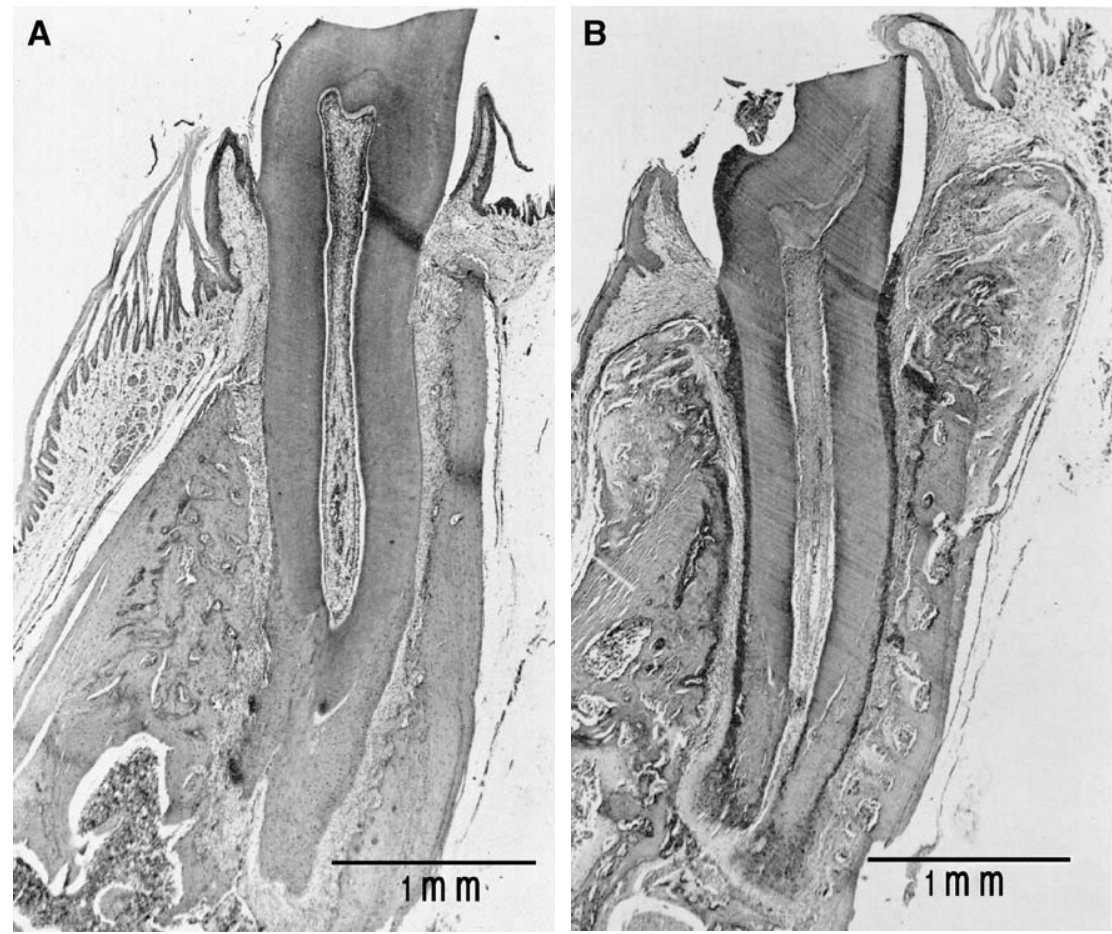

Fig. 13. Histopathologic pictures of molar teeth of a control rat $(A)$ and a rat given dihydrotachysterol showing hypercementosis, hyperosteoidosis, narrowing of the periodontal space, and bulbous enlargement of crestal alveolar bone with loss of biologic width (B). (From Glickman I, Selye H, Smulow JB. Reduction by calciphylaxis of the effects of chronic dihydrotachysterol overdosage upon the periodontium. J Dent Res 1965;44:735-6; with permission.)

buccal and lingual bone occurred most notably at muscle insertions [95]. Alveolar bone formation at the site of a gingival injury took place more rapidly and was more evident in experimentally injured than noninjured rats that also received DHT [96]. Similarly, mineralization of the periodontal ligament and gingival connective tissue was enhanced when a collagendamaging agent was given to rats receiving intraperitoneal injections of vitamin $\mathrm{D}_{2}$ [97]. In rats given DHT, degeneration of the periodontal ligament, hypercementosis, hyperosteoidosis, narrowing of the periodontal space, and ankylosis were markedly more pronounced in furcation areas [91,98] and teeth that were in occlusion [99] or subjected to traumatic occlusion [100]. Daily masticatory stress could be the reason why chronic increased vitamin D intake manifests sooner and is more pronounced in periodontal tissues compared with other soft tissues, and FORL may therefore occur before or without obvious signs of vitamin D-induced systemic disease. 

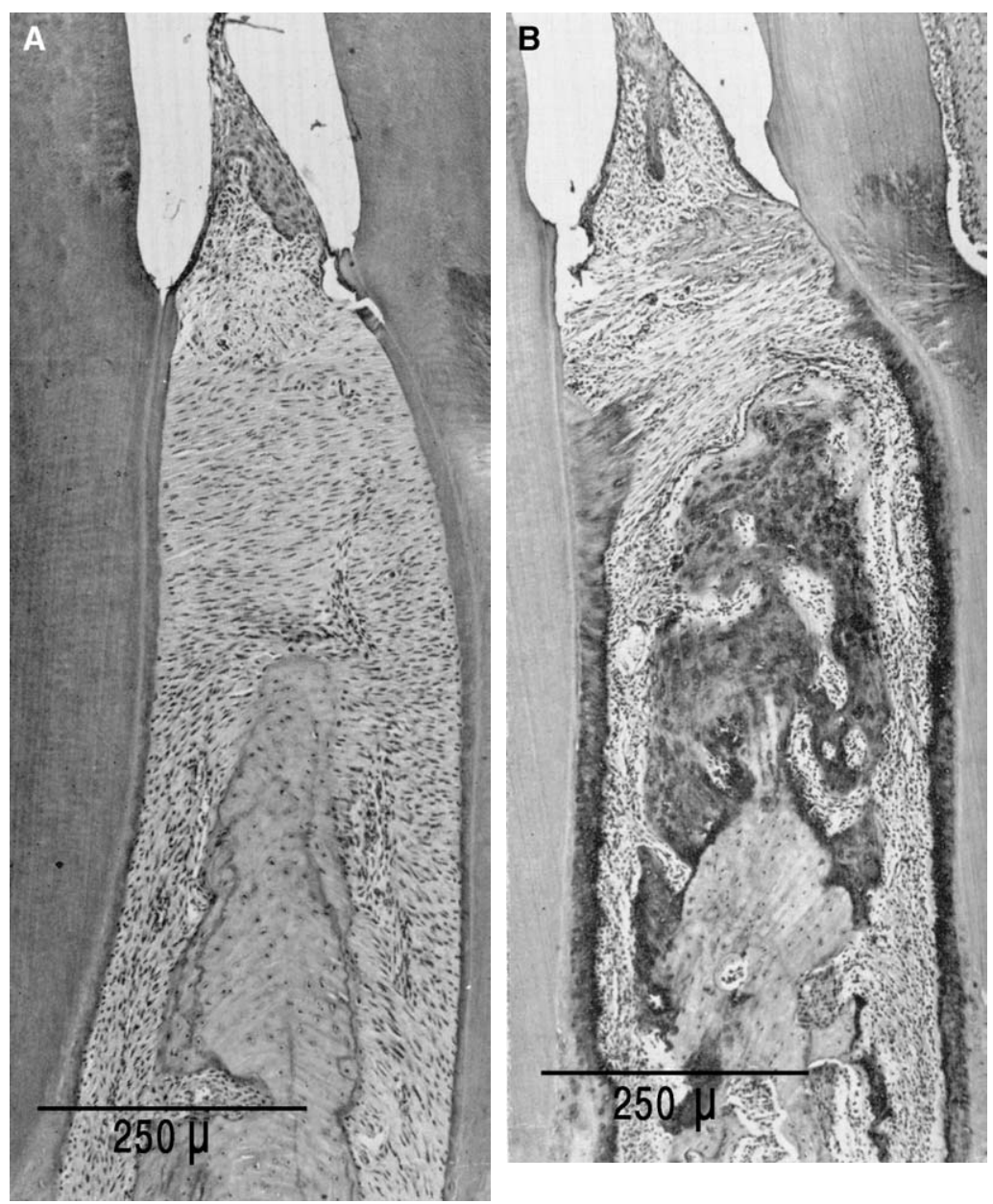

Fig. 14. Histopathologic pictures of interdental area from teeth of a control rat $(A)$ and a rat given dihydrotachysterol showing hypercementosis, hyperosteoidosis, edematous degeneration of the periodontal ligament, narrowing of the periodontal space, bulbous enlargement of crestal alveolar bone, coronal displacement of transeptal fibers, and reduction of biologic width $(B)$. (From Glickman I, Selye H, Smulow JB. Reduction by calciphylaxis of the effects of chronic dihydrotachysterol overdosage upon the periodontium. J Dent Res 1965;44:738; with permission.)

\section{Experimental studies with vitamin D and vitamin D metabolites}

Numerous reports describe the effects of excess vitamin D and vitamin D metabolites on the pulp-dentin complex and periodontium in experimental animals (rodents, lagomorphs, pigs, and dogs) (Table 1).

In the pulp-dentin complex, pulpal hyperemia and degeneration, decreased width of the predentin layer, and formation of osteodentin and 

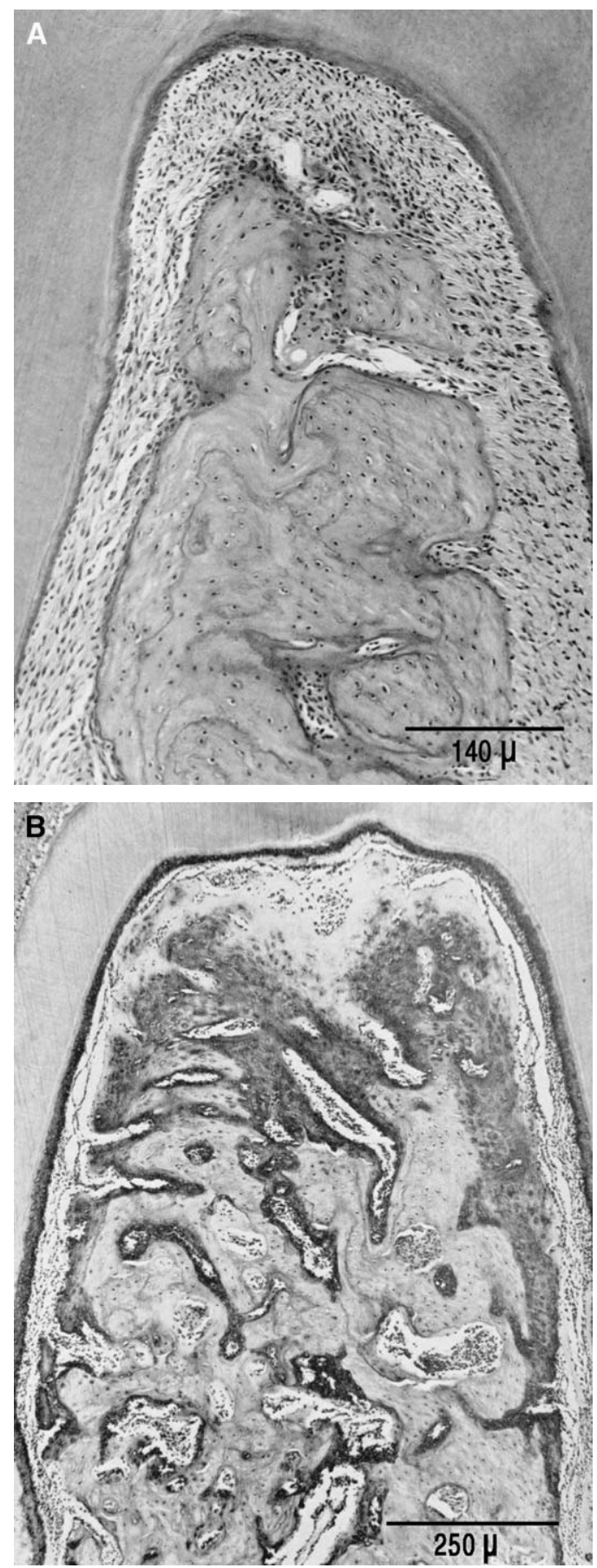

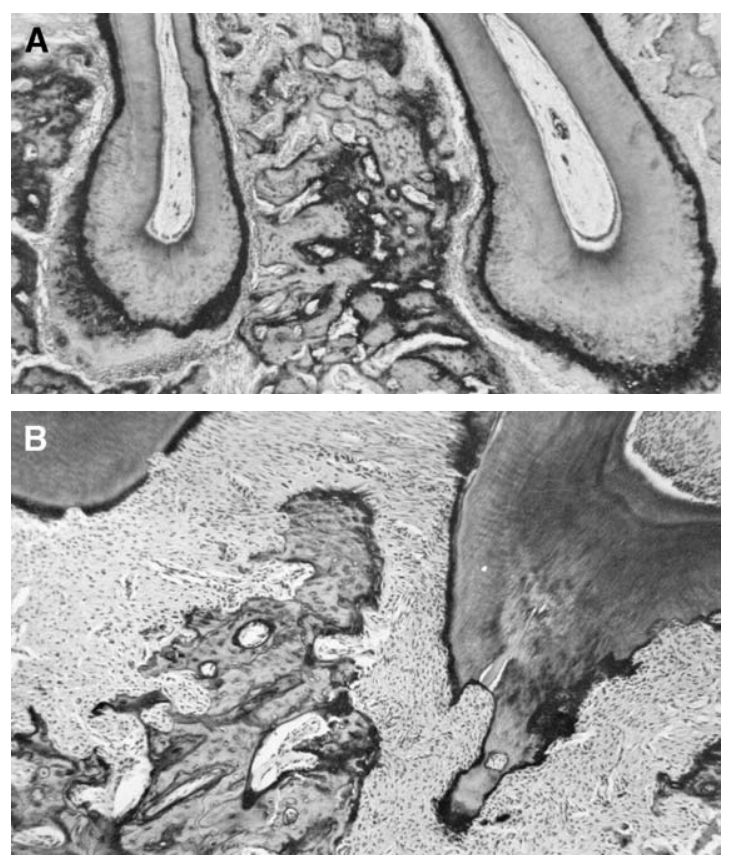

Fig. 16. Histopathologic pictures of rats given dihydrotachysterol showing bulbous enlargement of root apices $(A)$ and resorption of cementum, dentin, and alveolar bone $(B)$. (From Moskow BS, Baden E. The effect of chronic dihydrotachysterol overdosage on the tissues of the periodontium. Periodontics 1964;2:279-80; with permission.)

irregular dentin containing small vascular canals (Fig. 9) have been reported [101-107].

In the periodontium, periodontal ligament hyperemia, edema, and degeneration with fiber disorientation; mineralization of Sharpey's fibers; hypercementosis with abnormal thickening of cervical cementum and a bulbous appearance of root apices; hyperosteoidosis along periosteal and endosteal surfaces; reduced endosteal lumina; bone marrow fibrosis; bulbous enlargement of alveolar plates with coronal displacement of transeptal fibers at the alveolar margin; narrowing of the periodontal space; dentoalveolar ankylosis; granulation tissue formation; irregular resorptive lacunae in cementum and dentin; and a mixed pattern of osteoporosis and osteosclerosis (Fig. 10-16) have been reported [91,95-102,104,106-125].

Fig. 15. Histopathologic pictures of furcation area of molar teeth in a control rat $(A)$ and a rat given dihydrotachysterol showing hypercementosis, hyperosteoidosis, degeneration of the periodontal ligament, and narrowing of the periodontal space (B). (From Glickman I, Selye H, Smulow JB. Reduction by calciphylaxis of the effects of chronic dihydrotachysterol overdosage upon the periodontium. J Dent Res 1965;44:743-4; with permission.) 
Extrapolating these findings to the domestic cat should be done with caution, however, because the results of these experimental studies are not uniform. Furthermore, the ages, sizes, and species of animals; the character of their diets; the varying forms, quantities, and routes of administration of vitamin D and vitamin D metabolites; and the duration of the experiments differed. Nevertheless, there are distinct similarities between the changes in dental and periodontal tissues induced by administration of excess vitamin D and vitamin D metabolites in experimental animals and radiographic and microscopic features that can be found in teeth from cats with FORL (eg, thin predentin layer, irregular dentin formation, periodontal ligament degeneration and fiber disorientation, hypercementosis, hyperosteoidosis, thickening of crestal alveolar bone, narrowing of the periodontal space, dentoalveolar ankylosis, root resorption, mixed pattern of osteoporosis and osteosclerosis). Vitamin D-induced thickening of cervical cementum and abnormal apposition of osteoid at the alveolar crest and other periosteal surfaces causing bulbous enlargement of alveolar plates and coronal displacement of transeptal fibers could result in reduction of the biologic width (the dimension of space occupied by junctional epithelium and gingival connective tissue) and loss of gingival attachment. Supereruption of teeth in cats with increased vitamin D activity may actually be an attempt to maintain or re-establish normal biologic width.

Certain findings are worthy of additional discussion, including (a) differences in effects of vitamin D and vitamin D metabolites between continuously growing and continuously erupting teeth and between young and adult animals and (b) apparent alleviation of the detrimental effects of vitamin D and vitamin D metabolites by concurrent administration of other agents. In rats, pulpal mineralization and pulp stones occurred more commonly in incisors than in molars and more commonly in younger than in older animals [107], which may be an indication that vitamin D activity is more influential on "young" or continuously renewing tissue. Although pulpal mineralization has not been reported in permanent teeth of cats with FORL, pulp stones have been demonstrated in experimental vitamin D studies in puppies $[108,110,114]$. Young animals (dogs and rats) showed initial alveolar bone resorption and osteoporosis followed by hyperosteoidosis and osteosclerosis with a narrowing of endosteal spaces, whereas alveolar bone resorption and osteoporosis were predominant in adult or older animals [107,108]. Studies investigating the appearance of alveolar bone in younger and older FORL-affected cats have not yet been conducted. Effects of vitamin D or vitamin D metabolites were less severe or could be reduced in animals given various amounts of vitamin A [108,114], sexual hormones [95,117], ferric dextran [91,98,123], or sodium fluoride [118], in addition to excess administration of vitamin D or vitamin D metabolites. This may be of interest when considering future research that focuses on prevention of FORL. 


\section{Summary}

The following conclusions can be drawn:

1. Cats depend on dietary vitamin $\mathrm{D}$ intake because they are not able to produce vitamin $\mathrm{D}$ in the skin.

2. Some commercial cat foods contain vitamin D concentrations in excess of current maximal allowances.

3. Cats with FORL have significantly higher serum concentrations of 25OHD compared with cats without FORL, indicating that cats with FORL must have ingested higher concentrations of dietary vitamin D.

4. Cats with FORL have significantly decreased urine specific gravity compared with cats without FORL.

5. Experimental studies on laboratory animals have shown that excess administration of vitamin D or vitamin D metabolites causes changes to dental and periodontal tissues that resemble many characteristics of teeth from cats with FORL.

6. Clinical and experimental studies have shown that excess administration of vitamin D or vitamin D metabolites can lead to soft tissue mineralization and various degrees of renal disease.

Dietary intake of excess vitamin D over several years may lead to periodontal ligament degeneration, narrowing of the periodontal space, dentoalveolar ankylosis, and root replacement resorption. If such a process occurs close to the gingival margin, an inflammatory component may join the disease. Further histologic and experimental studies are required to determine the role of daily masticatory stresses on the development of FORL and to verify relations between FORL, vitamin D, and renal insufficiency.

\section{References}

[1] Hopewell-Smith A. The process of osteolysis and odontolysis, or so-called "absorption" of calcified tissues: a new and original investigation. Dental Cosmos 1930;72:1036-48.

[2] Reiter AM. Feline "odontolysis" in the 1920s: the forgotten histopathological study of feline odontoclastic resorptive lesions (FORL). J Vet Dent 1998;15:35-41.

[3] Kerebel B, Daculsi G. Histologie et histopathologie dentaires du chat. Sci Rech Odontostomatol 1971;7(5):29-32.

[4] Schneck GW, Osborn JW. Neck lesions in the teeth of cats. Vet Rec 1976;99:100.

[5] Reiter AM. The role of calciotropic factors in the etiology of feline odontoclastic resorptive lesions (FORL) [thesis]. Vienna: University of Veterinary Medicine Vienna; 2004.

[6] Reiter AM. Biology of alveolar bone and tooth resorbing cells. In: Proceedings of the 12th Annual Veterinary Dental Forum. New Orleans, 1998. p. 225-7.

[7] Sahara N, Toyoki A, Ashizawa Y, et al. Cytodifferentiation of the odontoclast prior to the shedding of human deciduous teeth: an ultrastructural and cytochemical study. Anat Rec 1996;244:33-49.

[8] Harvey CE, Orsini P, McLahan C, et al. Mapping the radiographic central point of feline dental resorptive lesions. J Vet Dent 2004;21:15-21. 
[9] Eriksen T, Koch R, Nautrup CP. Microradiography of the feline marginal periodontium with a microfocal high-resolution X-ray system. Scand J Dent Res 1994;102:284-9.

[10] Reiter AM, Mendoza K. Feline odontoclastic resorptive lesions. An unsolved enigma in veterinary dentistry. Vet Clin N Am Small Anim Pract 2002;32:791-837.

[11] Keinath G. Tierzahnheilkunde bei Katzen unter besonderer Beruecksichtigung der Aetiologie und Therapie von "Neck-Lesions" [thesis]. Munich: Munich University, Faculty of Human Medicine; 1997.

[12] Ohba S, Kiba H, Kuwabara M, et al. A histopathological study of neck lesions in feline teeth. J Am Anim Hosp Assoc 1993;29:216-20.

[13] Okuda A, Harvey CE. Etiopathogenesis of feline dental resorptive lesions. Vet Clin N Am Small Anim Pract 1992;22:1385-404.

[14] Reichart PA, Dürr U-M, Triadan H, et al. Periodontal disease in the domestic cat. A histopathologic study. J Periodont Res 1984;19:67-75.

[15] Roes F. Pathogenese, Diagnostik und Therapie bei "neck lesions" der Katze unter Verwendung von Glas-Ionomer-Zementen [thesis]. Berlin: Free University of Berlin, Faculty of Veterinary Medicine; 1996.

[16] Steinberg S. Histologische Untersuchungen zu Fruehveraenderungen der Felinen Odontoklastischen Resorptiven Laesionen (FORL) an klinisch gesunden Zaehnen [thesis]. Berline: Free University of Berlin, Faculty of Veterinary Medicine; 2002.

[17] Trope M, Chivian N. Root resorption. In: Cohen S, Burns RC, editors. Pathways of the pulp. 6th edition. St. Louis: Mosby-Year Book; 1994. p. 486-512.

[18] DuPont GA, DeBowes LJ. Comparison of periodontitis and root replacement in cat teeth with resorptive lesions. J Vet Dent 2002;19:71-5.

[19] Berger M, Schawalder P, Stich H, et al. "Neck Lesion" bei Grosskatzen; Untersuchungen beim Leoparden (Panthera pardus). Kleintierpraxis 1995;40:537-49.

[20] Schlup D, Stich H. Epidemiologische und morphologische Untersuchungen am Katzengebiß. II. Mitteilung: Morphologische Untersuchungen der "neck lesions". Kleintierpraxis 1982;27:179-88.

[21] George DI, Miller RL. Idiopathic resorption of teeth. A report of three cases. Am J Orthod 1986;89:13-20.

[22] Henry JL, Weinmann JP. The pattern of resorption and repair of human cementum. J Am Dent Assoc 1951;42:270-90.

[23] Shigeyama Y, Grove TK, Strayhorn C, et al. Expression of adhesion molecules during tooth resorption in feline teeth: a model system for aggressive osteoclastic activity. J Dent Res 1996;75:1650-7.

[24] Forsberg A, Lagergren C, Lönnerblad T. The periodontal tissue of mandibular premolars and molars in some mammals. A comparative anatomical study. Svensk TandlakareTidskrift 1969;62(Suppl 1):1-54.

[25] Gorrel C, Larsson A. Feline odontoclastic resorptive lesions: unveiling the early lesion. J Small Anim Pract 2002;43:482-8.

[26] Reiter AM. Etiopathology of feline odontoclastic resorptive lesions (FORL). In: Proceedings of the 12th Annual Veterinary Dental Forum. New Orleans, 1998. p. 228-34.

[27] Blackwood HJJ. Resorption of enamel and dentine in the unerupted tooth. Oral Surg Oral Med Oral Pathol Oral Radiol Endod 1958;11:79-85.

[28] Boyle PE. Cementum - changes with age, function, and infection. In: Kronfeld's histopathology of the teeth and their surrounding structures. Philadelphia: Lea \& Febiger; 1955. p. 258-72.

[29] Stafne EC, Austin LT. Resorption of embedded teeth. J Am Dent Assoc 1945;32:1003-9.

[30] Zemsky JL. Hypercementosis in relation to unerupted and malposed teeth. A preliminary report. J Dent Res 1931;11:159-74.

[31] Kupfer IJ. Correlation of hypercementosis with toxic goiter. A preliminary report. J Dent Res 1951;30:734-6. 
[32] Farmer ED, Lawton FE. The effects of endocrine disorders on the jaws and teeth. In: Stones' oral and dental diseases. Aetiology, histopathology, clinical features and treatment. 5th edition. Baltimore: Williams \& Wilkins; 1966. p. 30-48.

[33] Gardner BS, Goldstein H. The significance of hypercementosis. Dent Cosmos 1931;73: 1065-9.

[34] Schour I, Massler M. Endocrines and dentistry. J Am Dent Assoc 1943;30:595-603.

[35] Rushton MA. The dental tissues in osteitis deformans. Guys Hosp Rep 1938;88:163-71.

[36] Smith NHH. Monostotic Paget's disease of the mandible presenting with progressive resorption of the teeth. Oral Surg Oral Med Oral Pathol Oral Radiol Endod 1978;46: 246-53.

[37] Stafne EC. Paget's disease involving the maxilla and the mandible: report of a case. J Oral Surg 1946;4:114-5.

[38] Farmer ED, Lawton FE. Cementum: abnormalities associated with its formation. In: Stones' oral and dental diseases. Aetiology, histopathology, clinical features and treatment. 5th edition. Baltimore: Williams \& Wilkins; 1966. p. 447-67.

[39] King JD. Dietary deficiency, nerve lesions and the dental tissues. J Physiol 1937;88:62-77.

[40] Kellner E. Das Verhaeltnis der Zement- und Periodontalbreiten zur funktionellen Beanspruchung der Zaehne. Z Stomatol 1931;29:44-62.

[41] Kronfeld R. Die Zementhyperplasien an nicht funktionierenden Zaehnen. Z Stomatol 1927;25:1218-27.

[42] Itoiz MA, Carranza FA. The gingiva. In: Newman MG, Takei HH, Carranza FA, editors. Carranza's clinical periodontology. 9th edition. Philadelphia: WB Saunders; 2002. p. 16-35.

[43] Black GV. The periosteum and peridental membranes. Dent Rev 1887;1:233-43, 353-65.

[44] Lyon KF. Subgingival odontoclastic resorptive lesions. Classification, treatment and results in 58 cats. Vet Clin N Am Small Anim Pract 1992;22:1417-32.

[45] Carranza FA, Camargo PM. Periodontal response to external forces. In: Newman MG, Takei HH, Carranza FA, editors. Carranza's clinical periodontology. 9th edition. Philadelphia: WB Saunders; 2002. p. 371-83.

[46] Lukman K, Pavlica Z, Juntes P. Prevalence patterns and histological survey of feline dental resorptive lesions. In: Proceedings of the 8th Annual Scientific Meeting of the British Veterinary Dental Association. Birmingham, 1996.

[47] Boling LR. Blood vessels of the dental pulp. Anat Rec 1942;82:25-37.

[48] Winter GB, Kramer IRH. Changes in periodontal membrane and bone following experimental pulpal injury in deciduous molar teeth in kittens. Arch Oral Biol 1965;10: 279-89.

[49] Negro VB, Hernandez SZ, Maresca BM, et al. Furcation canals of the maxillary fourth premolar and the mandibular first molar teeth in cats. J Vet Dent 2004;21:10-4.

[50] Okuda A, Habata I. Lacunae or tubular structures in dentin of feline teeth. J Anim Res Found 1993;2(1):7-14.

[51] Okuda A, Harvey CE. Histopathological findings of features of odontoclastic resorptive lesions in cat teeth with periodontitis. In: Proceedings of the 5th Annual Veterinary Dental Forum. New Orleans, 1991. p. 141-4.

[52] Berger M, Stich H, Schaffner T, et al. Testimony from a silent late medieval witness-what can it tell us about FORL? In: Proceedings of the 13th European Congress of Veterinary Dentistry. Krakow, 2004. p. 17-8 (plus addendum).

[53] Dobbertin F. Zur Pathologie der Zahn- und Zahnbetterkrankungen bei Felis silvestris forma catus [thesis]. Hamburg: Hamburg University, Faculty of Human Medicine; 1993.

[54] Harvey CE, Alston WE. Dental diseases in cat skulls acquired before 1960. In: Proceedings of the 4th Annual Veterinary Dental Forum. Las Vegas, 1992. p. 41-3.

[55] DeLaurier A, Jackson B, Ingham K, et al. Biochemical markers of bone turnover in the domestic cat: relationships with age and feline osteoclastic resorptive lesions. J Nutr 2002; 132(Suppl):1742S-4S. 
[56] Clarke DE, Cameron A. Feline dental resorptive lesions in domestic and feral cats and the possible link with diet. In: Proceedings of the 5th World Veterinary Dental Congress. Birmingham, 1997. p. 33-4.

[57] How KL, Hazewinkel AW, Mol JA. Dietary vitamin D dependance of cat and dog due to inadequate cutaneous synthesis of vitamin D. Gen Comp Endocrinol 1994;96:12-8.

[58] National Research Council. Nutrient requirements of cats. Washington, DC: National Academy Press; 1986. p. 4, 15, 16, 23, 56.

[59] Morris JG, Earle KE. Growing kittens require less dietary calcium than current allowances. J Nutr 1999;129:1698-704.

[60] Morris JG, Earle KE, Anderson PA. Plasma 25-hydroxyvitamin D in growing kittens is related to dietary intake of cholecalciferol. J Nutr 1999;129:909-12.

[61] Morris JG. Vitamin D synthesis by kittens. Vet Clin Nutr 1996;3(3):88-92.

[62] Haruna A, Kawai K, Takaba T, et al. Dietary calcinosis in the cat. J Anim Clin Res Found 1992;1(1):9-16.

[63] Morita T, Awakura T, Shimada A, et al. Vitamin D toxicosis in cats: natural outbreak and experimental study. J Vet Med Sci 1995;57:831-7.

[64] Sato R, Yamagishi H, Naito Y, et al. Feline vitamin D toxicosis caused by commercially available cat food. J Jpn Vet Med Assoc 1993;46:577-81.

[65] Sih TR, Morris JG, Hickman MA. Chronic ingestion of high concentrations of cholecalciferol in cats. Am J Vet Res 2001;62:1500-6.

[66] Holick MF. Vitamin D: photobiology, metabolism, mechanism of action, and clinical applications. In: Favus MJ, editor. Primer on the metabolic bone diseases and disorders of mineral metabolism. 4th edition. Philadelphia: Lippincott Williams \& Wilkins; 1999. p. $92-8$.

[67] Itonaga I, Sabokbar A, Murray DW, et al. Effect of osteoprotegerin and osteoprotegerin ligand on osteoclast formation by arthroplasty membrane derived macrophages. Ann Rheum Dis 2000;59:26-31.

[68] Burgess TL, Qian Y-X, Kaufman S, et al. The ligand for osteoprotegerin (OPGL) directly activates mature osteoclasts. J Cell Biol 1999;145:527-38.

[69] Braem M, Lambrechts P, Vanherle G. Stress-induced cervical lesions. J Prosthet Dent 1992; 67:718-22.

[70] Lee WC, Eakle WS. Possible role of tensile stress in the etiology of cervical erosive lesions of teeth. J Prosthet Dent 1984;52:374-80.

[71] Lee WC, Eakle WS. Stress-induced cervical lesions: review of advances in the past 10 years. J Prosthet Dent 1996;75:487-94.

[72] Bevenius J, L'Estrange P, Karlsson S, et al. Idiopathic cervical lesions: in vivo investigation by oral microendoscopy and scanning electron microscopy. A pilot study. J Oral Rehabil 1993;20:1-9.

[73] Goel VK, Khera SC, Ralston JL, et al. Stresses at the dentinoenamel junction of human teeth. J Prosthet Dent 1991;66:451-9.

[74] Burke FJT, Johnston N, Wiggs RB, et al. An alternative hypothesis from veterinary science for the pathogenesis of noncarious cervical lesions. Quintessence Int 2000;31:475-82.

[75] Johnston N. Acquired feline oral cavity disease. Part 2: feline odontoclastic resorptive lesions. In Pract 2000;22:188-97.

[76] Orban B. Resorption and repair on the surface of the root. J Am Dent Assoc 1928;15: 1768-77.

[77] Chipps HD. Two cases of root resorption. Dental Cosmos 1928;70:461-2.

[78] Coolidge ED. The reaction of cementum in the presence of injury and infection. J Am Dent Assoc 1931;18:499-525.

[79] Wood P, Rees JS. An unusual case of furcation external resorption. Int Endod J 2000;33: 530-3.

[80] Yusof WZ, Ghazali MN. Multiple external root resorption. J Am Dent Assoc 1989;118: $453-5$. 
[81] Rawlinson A. Treatment of root and alveolar bone resorption associated with bruxism. Br Dent J 1991;170:445-7.

[82] Harris EF, Robinson QC, Woods MA. An analysis of causes of apical root resorption in patients not treated orthodontically. Quintessence Int 1993;24:417-28.

[83] Itoiz ME, Carranza FA, Cabrini RL. Histologic and histometric study of experimental occlusal trauma in rats. J Periodontol 1963;34:305-14.

[84] Kameyama Y. Histopathologic and autoradiographic studies of the changes of the rat periodontium in experimental traumatic occlusion. Bull Tokyo Med Dent Univ 1968;15:339-57.

[85] Orban B. Tissue changes in traumatic occlusion. J Am Dent Assoc 1928;15:2090-106.

[86] Ramfjord SP, Kohler CA. Periodontal reaction to functional occlusal stress. J Periodontol 1959;30:95-112.

[87] Mjör IA, Stenvik A. Microradiography and histology of decalcified human teeth following experimental intrusion; with emphasis on resorption. Arch Oral Biol 1969;14:1355-64.

[88] Bhaskar SN, Orban B. Experimental occlusal trauma. J Periodontol 1955;26:270-84.

[89] Lefkowitz W, Waugh LM. Experimental depression of teeth. Am J Orthod Oral Surg 1945; 31:21-36.

[90] Boyle PE. Tooth resorption. In: Kronfeld's histopathology of the teeth and their surrounding structures. Philadelphia: Lea \& Febiger; 1955. p. 273-96.

[91] Glickman I, Selye H, Smulow JB. Reduction by calciphylaxis of the effects of chronic dihydrotachysterol overdose upon the periodontium. J Dent Res 1965;44:734-49.

[92] Sindelka Z. Die Kalziphylaxie im Zahnmark. Stoma (Heidelb) 1968;21:101-9.

[93] Selye H, Grasso S, Padmanabhan N. Topical injury as a means of producing calcification at predetermined points with dihydrotachysterol (DHT). Proc Zool Soc 1960;13(1):1-3.

[94] Selye H, Jean P, Veilleux R. Role of local trauma in the production of cutaneous calcinosis by dihydrotachysterol. Proc Soc Exp Biol Med 1960;104:409-11.

[95] Ratcliff PA, Krajewski J. The influence of methyl testosterone on dihydrotachysterol intoxication as it affects the periodontium. J Oral Ther Pharmacol 1966;2:353-61.

[96] Stahl SS, Cohen C, Epstein B. The responses of injured and noninjured rat periodontal tissues to a single administration of dihydrotachysterol. Oral Surg Oral Med Oral Pathol Oral Radiol Endod 1967;23:531-7.

[97] Shoshan S, Pisanti S, Sciaky I. The effect of hypervitaminosis D on the periodontal membrane collagen in lathyritic rats. J Periodont Res 1967;2:121-6.

[98] Moskow BS, Baden E, Zengo A. The effects of dihydrotachysterol and ferric dextran upon the periodontium in the rat. Arch Oral Biol 1966;11:1017-26.

[99] Kojima M. Experimental study on the physio-pathological changes of periodontal tissues. Bull Stomatol Kyoto Univ 1969;9:101-40.

[100] Takano K, Watanabe Y, Fuzihashi H, et al. The histological changes of alveolar bone, cementum and periodontal fiber due to experimental progeria-like syndrome of the rat. J Jpn Assoc Periodontol 1981;23:357-66.

[101] Cai JJ. Effect of vitamin D overdosage on the tooth and bone development of rabbits. Chin J Stomatol 1992;27:296-9, 319.

[102] Daemmrich K. Experimentelle $\mathrm{D}_{3}$-Hypervitaminose bei Ferkeln. Zentrabl Veterinarmed A 1963;10:322-49.

[103] Irving TJ, Weinmann JP, Schour I, et al. Experimental studies in calcification. VIII. The effect of large doses of calciferol on the dentin of the incisor in normal and nephrectomized rats. J Dent Res 1949;28:362-8.

[104] Pitaru S, Blauschild N, Noff D, et al. The effect of toxic doses of 1,25-dihydroxycholecalciferol on dental tissues in the rat. Arch Oral Biol 1982;27:915-23.

[105] Tempestini O. Nuove ricerche sugli effetti sperimentali della somministrazione di una alta dose di vitamina $\mathrm{D}_{2}$ nei tessuti dentali e paradentali del ratto. Riv Ital Stomatol 1952;7: 373-410.

[106] Weinmann J. Experimentelle Untersuchung ueber die Wirkung grosser Dosen Vigantol auf Knochen und Zaehne. Klin Wochenschr 1929;8:841-2. 
[107] Weinmann J. Untersuchungen an Knochen und Zaehnen der Ratte bei Verfuetterung grosser Dosen D-Vitamin. Dtsch Mschr Zahnheilk 1933;51:577-603, 625-54.

[108] Becks H. Dangerous effects of vitamin D overdosage on dental and paradental structures. J Am Dent Assoc 1942;29:1947-68.

[109] Becks H, Collins DA, Axelrod HE. The effects of a single massive dose of vitamin $\mathrm{D}_{2}$ (Dstoss therapy) on oral and other tissues of young dogs. Am J Orthod Oral Surg 1946;32: 452-62.

[110] Becks H, Collins DA, Freytag RM. Changes in oral structures of the dog persisting after chronic overdoses of vitamin D. Am J Orthod Oral Surg 1946;32:463-71.

[111] Bernick S, Ershoff BH, Lal JB. Effects of hypervitaminosis D on bones and teeth of rats. Int J Vitam Nutr Res 1971;41:480-9.

[112] Fahmy H, Rogers WE, Mitchell DF, et al. Effects of hypervitaminosis D on the periodontium of the hamster. J Dent Res 1961;40:870-7.

[113] Harris LJ, Innes JRM. Mode of action of vitamin D. Biochem J 1931;25:367-90.

[114] Hendricks JB, Morgan AF, Freytag RM. Chronic moderate hypervitaminosis D in young dogs. Am J Physiol 1947;149:319-32.

[115] Hirukawa T. Effect of dihydrotachysterol on the periodontal tissues in rats. Aichi-Gakuin J Dent Sci 1990;28:367-87.

[116] Kondo M. The changes of parodontal tissues of rat, caused by the administration of dihydrotachysterol. Shigaku 1971;59:219-45.

[117] Mabuchi H. Experimental study on the effect of sexual hormones and calcification enhancing substances on growth of the alveolar bone. Bull Stomatol Kyoto Univ 1970;10: $31-60$

[118] Miwa Y, Watanabe Y. The effect of fluoride on the periodontal tissues of dihydrotachysterol administered rats. Bull Josai Dent Univ 1985;14:343-54.

[119] Morgan AF, Axelrod HE, Groody M. The effect of a single massive dose of vitamin $\mathrm{D}_{2}$ on young dogs. Am J Physiol 1947;149:333-9.

[120] Moskow BS, Baden E. The effect of chronic dihydrotachysterol overdosage on the tissues of the periodontium. Periodontics 1964;2:277-83.

[121] Nomura H. Histopathological study of experimental hypervitaminosis $D_{2}$ on the periodontium of the rat. Shikwa Gakuho 1969;69:539-93.

[122] Ratcliff PA, Itokazu H. The effect of dihydrotachysterol on the teeth and periodontium. J Periodontol 1964;35:320-5.

[123] Ratcliff PA, Itokazu $\mathrm{H}$. The effect of dihydrotachysterol and ferric dextran on the teeth and periodontium of the rat. J Oral Ther Pharmacol 1964;1:7-22.

[124] Takano K, Watanabe Y. The histological study on osteoid- and cementoid-like tissues of rats treated with dihydrotachysterol. Bull Josai Dent Univ 1987;16:307-22.

[125] Terai Y. Studies on experimental stimulation of ossification in the paradental tissues of rats and blood level of Ca, P and alkaline phosphatase in them. Bull Stomatol Kyoto Univ 1968; 8(4):191-244. 